Journal for

ImmunoTherapy of Cancer

\section{IL-6 modulation for COVID-19: the right patients at the right time?}

To cite: Ascierto PA, Fu B, Wei H. IL-6 modulation for COVID-19: the right patients at the right time? Journal for ImmunoTherapy of Cancer 2021;9:e002285. doi:10.1136/ jitc-2020-002285

Accepted 23 March 2021

\section{Check for updates}

C Author(s) (or their employer(s)) 2021. Re-use permitted under CC BY-NC. No commercial re-use. See rights and permissions. Published by BMJ.

${ }^{1}$ Unit of Melanoma, Cancer Immunotherapy and Development Therapeutics, Istituto Nazionale Tumori IRCCS

Fondazione Pascale, Napoli, Italy ${ }^{2}$ Institute of Immunology and the CAS Key Laboratory of Innate Immunity and Chronic Disease, School of Life Science and Medical Center; Department of Pathology, The First Affiliated Hospital of USTC, Division of Life Sciences and Medicine, University of Science and Technology of China, Hefei, China

Correspondence to Dr Paolo Antonio Ascierto; paolo.ascierto@gmail.com

\begin{abstract}
The ongoing pandemic caused by the novel coronavirus SARS-CoV-2 has disrupted the global economy and strained healthcare systems to their limits. After the virus first emerged in late 2019, the first intervention that demonstrated significant reductions in mortality for severe COVID-19 in large-scale trials was corticosteroids. Additional options that may reduce the burden on the healthcare system by reducing the number of patients requiring intensive care unit support are desperately needed, yet no therapy has conclusively established benefit in randomized studies for the management of moderate or mild cases of disease. Severe COVID-19 disease is characterized by a respiratory distress syndrome accompanied by elevated levels of several systemic cytokines, in a profile that shares several features with known inflammatory pathologies such as hemophagocytic lymphohistiocytosis and cytokine release syndrome secondary to chimeric antigen receptor (CAR) T cell therapy. Based on these observations, modulation of inflammatory cytokines, particularly interleukin (IL)-6, was proposed as a strategy to mitigate severe disease. Despite encouraging recoveries with anti-IL-6 agents, especially tocilizumab from single-arm studies, early randomized trials returned mixed results in terms of clinical benefit with these interventions. Later, larger trials such as RECOVERY and REMAP-CAP, however, are establishing anti-IL-6 in combination with steroids as a potential option for hypoxic patients with evidence of hyperinflammation. We propose that a positive feedback loop primarily mediated by macrophages and monocytes initiates the inflammatory cascade in severe COVID-19, and thus optimal benefit with anti-IL-6 therapies may require intervention during a finite window of opportunity at the outset of hyperinflammation but before fulminant disease causes irreversible tissue damage - as defined clinically by $C$ reactive protein levels higher than $75 \mathrm{mg} / \mathrm{L}$.
\end{abstract}

\section{INTRODUCTION}

More than a year since the first case report of an atypical pneumonia caused by the novel coronavirus SARS-CoV-2 the COVID-19 pandemic continues to rage unabated, surpassing 1.5 million deaths worldwide in early December 2020. ${ }^{1}$ The case fatality rate varies by region, with the most deaths occurring in people over 70 years of age. ${ }^{2}$ Even though the mortality rate in the USA is less than $2 \%$, almost 200,000 excess deaths were attributed to COVID-19 through October,
$2020,{ }^{3}$ and current trends indicate that the human toll will only continue to increase. Although vaccines showed promising results in topline results from phase III trials reported in late 2020, distribution infrastructure is lacking, and several months may still elapse before a sufficient proportion of the population is inoculated to achieve herd immunity. COVID-19 is an ongoing emergency for the global community, and it is imperative to identify treatments to alleviate the burden on the healthcare system. Furthermore, although COVID-19 has disrupted all aspects of modern life, the pandemic also has important consequences for cancer caredelaying or interrupting treatments, halting clinical trials, and dramatically reducing routine screening. ${ }^{4}$ Conflicting evidence has emerged whether cancer was associated with worse outcomes, ${ }^{56}$ or if increased mortality among patients with malignancies is due to covariant factors such as smoking. ${ }^{7}$ Regardless, effective therapies for COVID-19 that may be integrated into anticancer regimens are needed.

The primary driver of organ damage in COVID-19 is an ongoing area of investigation, but likely a combination of direct tissue necrosis due to uncontrolled viral replication as well as the fulminant systemic inflammatory pathology and an accompanying coagulopathy instigated by SARS-CoV-2 infection both contribute to severe disease. Across studies, the most consistent predictors of mortality in COVID-19 are advanced age, male sex, and the presence of comorbidities such as diabetes, hypertension, and obesity. ${ }^{8-16} \mathrm{~A}$ recent pair of papers showed that defective interferon (IFN) signaling is associated with severe disease-either through inborn variation at key loci or the presence of autoantibodies to type I IFNs. ${ }^{17}$ Additionally elevated neutrophil to lymphocyte (NLR) ratio, high serum $\mathrm{C}$ reactive protein (CRP), and increased interleukin (IL) 6 have consistently been linked to severe disease. ${ }^{19-23}$

COVID-19 pneumonia is characterized by an acute respiratory distress syndrome 
(ARDS), accompanied by a hypercytokinemia with similarities to cytokine release syndrome (CRS) secondary to chimeric antigen receptor (CAR) $\mathrm{T}$ cell therapy as well as hemophagocytic lymphohistiocytosis (HLH)/macrophage activation syndrome (MAS). ${ }^{24-29}$ Based on the observed inflammatory pathology, immune-modulatory strategies, especially targeting IL-6, were proposed as a potential intervention to break the inflammatory cycle. ${ }^{30}$ Although uncontrolled studies seemed to demonstrate miraculous recoveries with anti-IL-6 therapies, randomized trials returned more mixed results. It is becoming clear that IL-6 modulation in COVID-19 is not a "magic bullet." However, some patients may benefit with antiIL-6, emphasizing a need for readily available biomarkers to identify candidates for intervention. This review will contextualize the results of the published accounts of anti-IL-6 therapy for COVID-19 through the lens of the monocyte-driven and macrophage-driven hyperinflammatory pathology. Extrapolating from published trials and mechanism-based insights into the pathology of the hyperinflammatory state, a potential "window of opportunity" is defined, clinically identifiable as CRP levels above $75 \mathrm{mg} / \mathrm{L}$, during which cytokine modulation may provide maximal benefit.

\section{CLINICAL COURSE AND INFLAMMATORY PATHOLOGY OF SARS- COV-2 INFECTION}

COVID-19 may present as a silent asymptomatic infection, a mild upper-respiratory tract illness, or severe disease characterized by fulminant inflammation, systemic coagulopathies, and severe damage to the lungs and cardiovascular system. ${ }^{2} 81631-34$ Evidence is also emerging that SARS-CoV-2 infection may occasionally cause long-term and sometimes debilitating symptoms, including fatigue, dyspnea, joint pain, and chest pain. ${ }^{35}$ Additionally, a delayed-onset inflammatory vasculitis with features akin to Kawasaki shock syndrome has been reported in children whose primary disease was asymptomatic or minimally symptomatic. ${ }^{36}$ Much remains unknown about the mechanisms responsible for the dramatic variations in disease presentation and long-term sequalae. However, since the first reported cases emerged in 2019, the infectious disease and immunology communities have gained some insight into correlates of severe disease and mortality.

Pulmonary failure secondary to ARDS is the primary cause of death in severe COVID-19. ${ }^{8} 143338$ Almost all cases of severe COVID-19 present with bilateral lung involvement, with characteristic ground glass opacities visible on CT imaging. ${ }^{34}{ }^{39}$ Multiple phenotypes on CT imaging have been described, ${ }^{40} 41$ and the different patterns correlate with disease severity. The signature findings in severe COVID-19 pneumonia, namely, intraalveolar edema, fibrin, and variable cellular infiltrates with a hyaline membrane, have been shown to associate with systemic inflammation, including elevated creatine kinase levels, increased neutrophil percentage, and high serum CRP. ${ }^{42}$ Autopsies have revealed pronounced mononuclear inflammatory infiltrates in the lungs, ${ }^{8} 4344$ microthrombi present in alveolar microvasculature as well as vessels of the heart, liver and kidneys, ${ }^{45}{ }^{46}$ as well as evidence of hemophagocytosis in the bone marrow. ${ }^{47} 48$ $\mathrm{T}$ cell counts are dramatically reduced in severe cases, and high lymphocyte expression of PD-1 is seen, which increases along with TIM-3 as disease severity progresses. ${ }^{49}$

\section{SARS-CoV-2 tissue tropism and pathological features of infection}

Angiotensin-converting enzyme 2 (ACE2) is the major entry receptor for SARS-CoV-2. ${ }^{50}{ }^{51}$ Emerging evidence suggests the virus may be able use additional targets, such as transferrin,${ }^{52}$ however, the biological relevance of alternate entry receptors remains unknown. Cell surface engagement occurs through the receptor binding domain of the SARS-CoV-2 spike protein, and cleavage by host proteases, including TMPRSS2, lysosomal cathepsins, and furin, is essential for viral entry. ${ }^{50} 5153$ ACE2 mRNA is found in almost all cell types, with especially high levels in renal, cardiovascular, and gastrointestinal system tissues, ${ }^{54}$ whereas protein expression is predominant on lung alveolar epithelial cells, enterocytes of the small intestine, as well as arterial and venous endothelial cells and arterial smooth muscle cells. ${ }^{55}$ ACE2 is a key component of the renin-angiotensin-aldosterone system (RAAS) as the catalytic enzyme that cleaves angiotensin II (Ang II) into angiotensins $1-7\left(\mathrm{Ang}_{1-7}\right) \cdot{ }^{56} \mathrm{In}$ addition to the well-established role of the RAAS in maintaining blood vessel tone, Ang II modulates numerous responses in local tissues, such that disruptions in ACE2 are associated with a number of inflammatory conditions, including atherosclerosis, heart failure, and chronic kidney disease. $^{57}$

The potential tissue tropism of SARS-CoV-2 is vast given the widespread distribution of its entry receptors throughout the body, and possibly even further expanded through spike protein pre-activation by furin to overcome a requirement for cell-surface TMPRSS2. ${ }^{51}$ SARS-CoV-2 viral particles have been observed in renal, intestinal, cardiac, and lymphoid tissues. ${ }^{58}$ SARS-CoV-2 has been demonstrated to directly infect type 2 pneumocytes and ciliated airway epithelial cells, ${ }^{48560}$ and viral RNA persists in lung endothelium and pneumocytes for several weeks after diagnosis. ${ }^{61}$ Cytopathic effects underlie some of the diffuse alveolar damage found in the lungs of patients with severe COVID-19. However, mononuclear inflammatory infiltrates dominated by monocytes and macrophages and lacking eosinophils are also characteristic of severe disease. ${ }^{843462-64}$ Although it is difficult to untangle the relative contributions of uncontrolled viral replication and aberrant immune reactions on COVID-19 mortality, it is becoming apparent that a damaging inflammatory pathology initiated in the lower respiratory tract eventually causes systemic tissue damage, disseminated coagulation, and multi-organ failure. 


\section{Cytokine storm in COVID-19}

"Cytokine storm" is a catch-all term that has used to describe a range of dysregulated immune reactions with a variety of instigating events ranging from sepsis to stem cell transplant. ${ }^{24}$ In recent years, the term has come to most commonly be associated with CRS after CAR T cell therapy-defined clinically by fever, hypoxia, and hypovolemia ${ }^{65}$ and characterized by profoundly elevated serum levels of CRP, IL-6, IL-10, and IFN $\gamma \cdot{ }^{66-69}$ CAR T cell-associated CRS shares several features with secondary HLH/MAS, in which serum levels of IFN- $\gamma$, tumor necrosis factor (TNF)- $\alpha$, IL-1 $\beta$, IL-2, IL-6, IL-12, IL-16, and IL-18 are elevated. ${ }^{70}$ Even though the levels of IL-6 measured in patients infected with SARS-CoV-2 are on the order of 10-fold lower than those seen in patients treated with CAR T cells, the hypercytokinemia seen with severe COVID-19, CRS, and HLH/MAS substantially overlap. ${ }^{825}$

The incidence of primary HLH, an autosomal recessive disorder, is roughly 1 in 100,000 live births. Secondary, or reactive, HLH/MAS may be triggered by infections (especially Epstein-Barr virus, CMV and HIV), autoimmune diseases, and some cancers. As many as $1 \%$ of patient with hematological cancers develop HLH, and mortality rates associated with this presentation approach $80 \%{ }^{72}{ }^{73}$ Viral HLH occurs when persistence of infected cells causes cytotoxic T lymphocytes and NK cells to continuously secrete inflammatory cytokines thus activating macrophages and resulting in a vicious cycle of IL-6 secretion, activation and an out-of-control positive feedback loop. ${ }^{70}$
Although IL-6 is essential for both innate and adaptive immunity, uncontrolled secretion leads to inflammatory damage to host cells. ${ }^{74}$ In severe COVID-19, IL-6 has been shown to be central among a network of 12 cytokines that are elevated in cases with poor prognoses. ${ }^{75}$

Although the presence of a cytokine storm in COVID-19 has been demonstrated many times over, the definition of "hyperinflammation" has varied substantially. Multiple studies have set out to define the threshold levels of various laboratory parameters that are associated with severe outcomes or mortality, and some meta-analyses have attempted to define threshold levels with prognostic power. ${ }^{76}$ Because IL-6 measurements are not readily available in many centers, however, cytokine measurements have limited utility for decision-making. Hyperinflammation at admission defined as CRP $>150 \mathrm{mg} / \mathrm{L}$ or doubling within 24 hours from greater than $50 \mathrm{mg} / \mathrm{L}$, or a ferritin concentration greater than $1500 \mu \mathrm{g} / \mathrm{L}$ was shown significantly increase the risk of next-day escalation of respiratory support or death (hazard ratio (HR) 2.24; $95 \%$ CI 1.62 to 287 ) in one cohort. ${ }^{77}$ Additionally, a six-criteria clinically based scoring criteria for the COVID-19 hyperinflammatory state (cHIS) has been proposed and validated, ${ }^{78}$ which shares several parameters with the scoring system for secondary HLH. ${ }^{79}$ The cHIS and HLH scores are summarized in table 1.

Viral HLH/MAS represents an overexuberant failsafe mechanism when primary protection measures do not clear infection. A similar process may be occurring in the

Table 1 Comparison of scoring systems for COVID-19 hyperinflammation and HLH

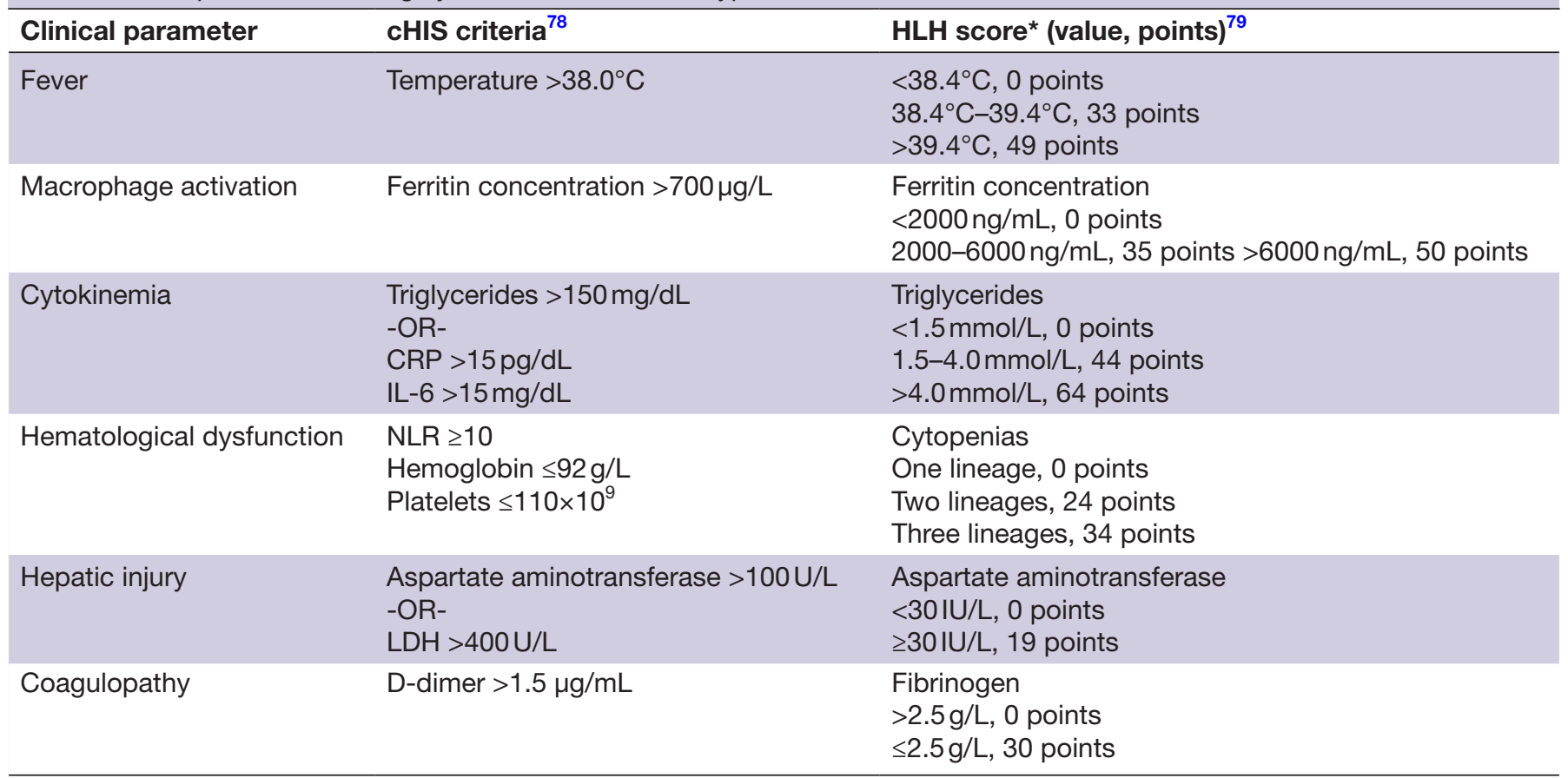

*HLH score also assigns points for organomegaly (23 for hepatomegaly or splenomegaly and 38 for hepatomegaly and splenomegaly), hemophagocytosis on bone marrow aspirate ( 0 if no and 35 if yes), and known immunosuppression such as long-term steroids or HIV ( 0 if no and 18 if yes).

cHIS, COVID-19 hyperinflammatory state; CRP, C reactive protein; HLH, hemophagocytic lymphohistiocytosis; LDH, lactate dehydrogenase; NLR, neutrophil to lymphocyte ratio. 
COVID-19 cytokine storm. Recent evidence for defective type I IFN signaling as risk factor for severe disease in COVID- $19^{1718}$ further underscores the notion that a failure of fist-line antiviral defenses leads to poor outcomes. Coronaviruses, like many other viruses have been shown to directly promote IL-6 production in immune and epithelial cells. ${ }^{80} 81$ Although the hyperinflammation in COVID-19 ARDS eventually affects the entire body, accumulating evidence implicates the myeloid lineage, specifically monocytes and macrophages, in driving severe disease. ${ }^{638283}$

\section{Contribution of monocytes and macrophages to COVID-19 inflammatory pathology}

Multiple mechanisms have been proposed to account for the hyperinflammatory state seen in severe COVID19. Pyroptotic death of infected cells, leading to release of IL-1 $\beta$ along with danger-associated molecular patterns could contribute to inciting an inflammatory cascade. ${ }^{84}$ Additionally, stem cell-derived type 2 alveolar cells infected with SARS-CoV-2 have been shown in vitro to upregulate expression of NF- $\mathrm{\kappa B}$ target genes, including IL6, CXCL8, CXCL2, CXCL3, CXCL10, and CXCL11. ${ }^{85}$ The viral spike protein itself has been proposed to possess superantigenicity, potentially inciting systemic cytokine release through major histocompatibility complex (MHC)-independent T cell activation. ${ }^{86} 87$

Emerging evidence, however, suggests a prominent role for monocytes and macrophages in the hyperinflammatory pathology that causes COVID-19 ARDS. Patients with severe COVID-19 have dramatically reduced numbers of $\mathrm{CD}^{+}$and $\mathrm{CD} 8^{+} \mathrm{T}$ cells, especially those requiring intensive care unit (ICU) care, and lymphocyte counts negatively correlate with levels of serum IL-6, IL-10, and TNF- $\alpha{ }^{49}$ Monocytes have been shown upregulate expression of IL-6 in response to spike protein from 2003 pandemic SARS-CoV. ${ }^{81}$ Monocyte-derived IL-6 has been also shown to drive CRS after CAR T cell therapy. ${ }^{88}$ Alveolar macrophages potently produce IL-6, and epithelial cells upregulate its expression in response to interferon gamma and danger-associated molecular patterns. ${ }^{89}$ Additionally, macrophages and dendritic cells express ACE2, ${ }^{63} 90$ and interactions with the virus may contribute to the generation of an inflammatory milieu.

SARS-CoV-2 binding to ACE2 on monocytes and macrophages leads to receptor internalization, ${ }^{91}$ thus enhancing local Ang II and vascular permeability. In the lungs, $\mathrm{CD} 14^{+} \mathrm{CD} 16^{+}$monocytes produce high levels of $\mathrm{Ang}_{1-7}$ and are thought to be vasoprotective. ${ }^{74}$ Loss of surface ACE2 on infection could therefore promote local endothelial dysfunction. Activation of the RAAS in monocytes plays a well-established role in the inflammatory and coagulation pathology in acute coronary syndromes. ${ }^{92}$ Ang II also promotes reactive oxygen species production and the expression of proinflammatory chemokines, leading to local accumulation of immune cells. ${ }^{93}$ The monocyte compartment is extensively remodeled in COVID-19, with phenotypic shifts in both circulating and tissue-resident populations. Although the original insult of SARS-CoV-2 infection in the lungs may initially target the epithelium, a resulting increased cytokine production by macrophages and perturbations to the RAAS initiate the cascade that leads to ARDS. A model for a monocytic and macrophage-driven inflammatory cascade originating in the alveoli is illustrated in figure 1.

Despite originating in the lungs, the inflammatory cascade set off by SARS-CoV-2 affects immune and stromal cells throughout the body. Elevated fractions of $\mathrm{CD} 14^{+} \mathrm{CD} 16^{+}$inflammatory monocytes with the capability to secrete granulocyte-macrophage colony-stimulating factor (GM-CSF) and high IL-6 expression have been measured in the peripheral blood of patients with COVID-19. ${ }^{83}$ Also seen is an increase in circulating Th17 cells in patients with severe disease ${ }^{44}$ Eosinopenia, which has now been linked with worse outcomes and poor responses to antivirals as well as to anti-IL-6 for COVID$19,{ }^{6294-96}$ is a well-established signature of inflammatory respiratory pathology. ${ }^{97} 98$ Eosinophils also play a role in the resolution of inflammation through their secretion of IL-4 and IL-14 along with a variety of lipid-based mediators of macrophage activity, ${ }^{99}$ suggesting that a failure of type 2 immunity to resolve inflammatory monocyte and macrophage activity may also contribute to the uncontrolled inflammation in COVID-19.

\section{RATIONALE FOR TARGETING IL-6}

Elevated IL-6 has consistently predicted mortality and severe outcomes in COVID-19. ${ }^{8} 1920100$ IL-6 signaling primarily occurs through the Janus kinase (JAK)/signal transducer and activator of transcription (STAT) pathway, predominantly via STAT3, which induces acute phase proteins, including CRP, amyloid A, and fibrinogen, and promotes differentiation, recruitment, survival and transformation programs in $\mathrm{T}$ and $\mathrm{B}$ cells and myeloid cells. ${ }^{101-103}$ JAK also modulates cellular survival through a phosphorylation cascade involving phosphorylates phosphatidylinositol-4,5-bisphosphate (PIP2), PI3K, phosphati-dylinositol-3,4,5-trisphosphate (PIP3), and $\mathrm{PkB} /$ Akt serine/threonine kinase. IL-6 signaling may also occur through RAS, RAF, and mitogen-activated protein kinases, culminating in acute phase protein generation. ${ }^{70}$ IL-6 is positively regulated by NF-kB, CAAT/enhancerbinding protein beta $(\mathrm{C} / \mathrm{EBP} \beta)$, specificity protein 1 (Sp1), cyclic AMP (cAMP) response element-binding protein (CREB), IFN regulatory factor 1 (IRF-1), activator protein 1 (AP-1), and TNF- $\alpha .{ }^{101}$ A negative feedback loop modulates IL-6 signaling through JAK/STAT, as STAT3 upregulates SOCS1 and SOCS3, which directly inhibit the catalytic activity of JAK ${ }^{69}$ Members of the Coronaviridae family may be especially likely to trigger dysregulated IL-6 production, as the 2003 pandemic SARS-CoV not only causes increased IL-6 production in epithelium compared with several influenza strains, but also induces lower levels of expression of the negative regulator SOCS3. ${ }^{104}$ 

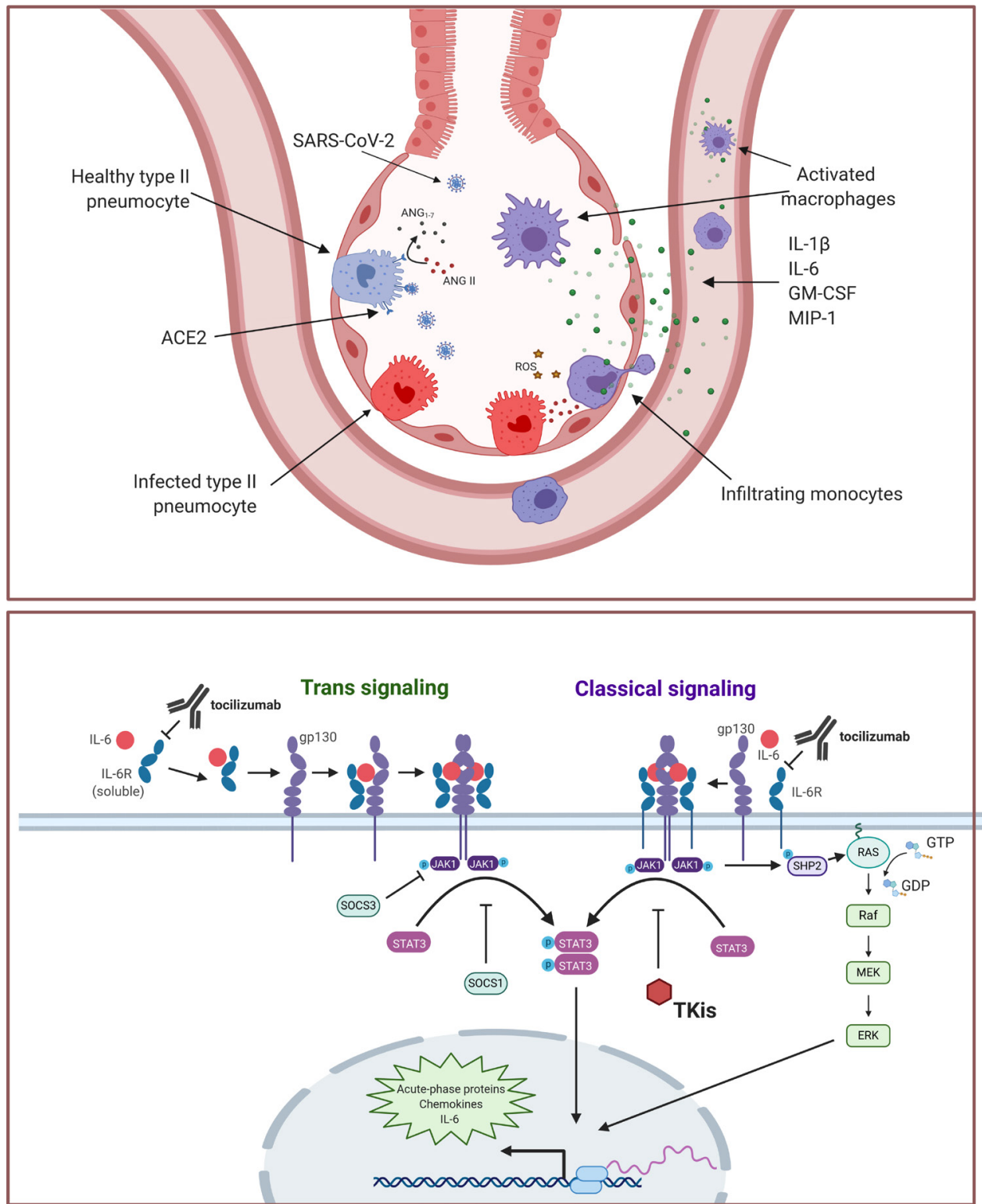

Figure 1 (A) Monocytic and macrophage contribution to SARS-CoV-2 hyperinflammation. SARS-CoV-2 directly infects pulmonary pneumocytes, causing cell death and the release of danger-associated molecular patterns, activating macrophages. Viral spike protein also triggers release of inflammatory cytokines, including interleukin (IL)-1 $\beta$, IL-6, granulocyte-macrophage colony-stimulating factor (GM-CSF) and macrophage inflammatory protine (MIP)-1 by alveolar-resident macrophages, promoting monocytic infiltration. Additionally, internalization of ACE2 on viral entry leads to increased angiotensin II, facilitating reactive oxygen species production, tissue damage, and an NF-KB-driven inflammatory gene expression program that includes production of chemokines, cytokines, and IL-6. (B) Contribution of IL-6 signaling to COVID-19 inflammatory cascade. Trimeric complex formation with IL-6, IL-6R and gp130 leads to dimerization and autophosphorylation of JAK1. Phosphorylated JAK1 triggers STAT3 phosphorylation and translocation to the nucleus, initiating acute-phase protein production, including chemokines, cytokines and IL-6. Phospho-JAK1 may also contribute to acute-phase transcription through an extracellular signal-regulated kinase (ERK)-dependent pathway that relies on SHP2 and Ras. Notably, IL-6 also induces expression of SOCS1 and SOC3, which negatively regulate JAK/STAT signaling. The classical signaling pathway is largely restricted to the lymphocyte compartment, where it contributes to adaptive immunity, whereas trans signaling may occur in any tissue type and is generally pro-inflammatory. Interventions that may alleviate inflammatory IL-6 signaling (ie, tocilizumab or tyrosine kinase inhibitors) are shown.

IL-6 is a highly pleiotropic cytokine with the potential to perturb almost all tissues in the body. Signaling through IL-6 in the central nervous system leading to prostaglandin production has been shown to underly fever under conditions of inflammation. ${ }^{105}$ Although the membrane-bound form of the IL-6 receptor (IL-6R) is expressed primarily on hepatocytes, megakaryocytes, and leukocytes ${ }^{103}$ both alternative splicing and cleavage by the membrane metalloproteases ADAM10 and ADAM17 may generate a soluble form. ${ }^{103} 106107$ Signaling requires assembly trimeric complex of IL-6, IL-6R, and the ubiquitously expressed 130-kilodalton signal-transducing 
b-receptor glycoprotein (gp130, also called CD130). 101102 IL-6 activity through the membrane-bound form of the receptor (termed classical signaling) is generally involved in wound healing and the resolution of inflammation, whereas the interaction between the soluble IL-6R/IL-6 complex and gp130 (trans signaling) is generally considered to be pro-inflammatory. ${ }^{101-103} 107$

Cytokine modulation for the treatment of hyperinflammation has become an established paradigm for several autoimmune disorders such as rheumatoid arthritis and colitis, and immuno-oncologists have readily adopted some anti-inflammatory agents originally developed for the rheumatology field for the treatment of immunerelated adverse events. For example, the TNF- $\alpha$ inhibitor infliximab is commonly used for the management of colitis secondary to checkpoint inhibitor therapy, ${ }^{108}$ and the IL-6R antagonist tocilizumab was approved by the Food and Drug Administration (FDA) for the treatment of CRS secondary to CAR T cell therapy in 2017 in a companion regulatory decision with tisagenlecleucel. ${ }^{109}$ Tocilizumab has also been used to treat some cases of checkpoint inhibitor-mediated pneumonitis. ${ }^{110}$ Administration of tocilizumab not only has anti-inflammatory properties, but the agent improves endothelial function leading to an increase of effective myocardial work through a reduction of inflammatory burden and oxidative stress. $^{111}$

Based on the central role for IL-6 in the inflammatory pathology of COVID-19 $9^{75}$ and a consistent association between elevated levels of the cytokine and severe disease outcomes, ${ }^{1022} 2325112113$ cytokine-modulatory therapies were proposed as a strategy to break the cycle of hyperinflammation. Reports from China ${ }^{28} 114$ and Italy, ${ }^{96} 115$ regions that were among the hardest-hit during the early months of 2020, seemed to demonstrate miraculous recoveries with anti-IL-6 therapies. Results of subsequent large-scale, randomized trials, however, were mixed, indicating that although IL-6 modulation for the management of COVID-19 may be useful in some circumstances, not all patients will benefit.

\section{OUTCOMES OF ANTI-IL-6 INTERVENTIONS ON COVID-19}

During the initial months of the pandemic, a case series that included 21 severe and critical patients from The First Affiliated Hospital of University of Science and Technology of China (Anhui Provincial Hospital) and Anhui Fuyang Second People's Hospital treated with tocilizumab in early February, 2020, demonstrated fever reduction within the first day after administration, and improvements of other symptoms within 5 days, with 15 of 20 patients $(75.0 \%)$ lowering $\mathrm{O}_{2}$ intake, radiographic improvement in 19 patients $(90.5 \%)$, a return to normal ranges of lymphocyte percentages in peripheral blood in 10 of 19 patients (52.6\%), and significantly decreased CRP in 16 of 19 patients $(84.2 \%) .{ }^{28}$ The single-arm TOCIVID-19 study enrolled 300 patients in Italy within 24 hours its launch, and subsequently concluded that tocilizumab reduced 14-day and 30-day mortality compared with a priori expected rates. ${ }^{116}$ Spurred on by these encouraging findings, numerous subsequent reports emerged of rapid and sustained recoveries in patients with severe COVID-19 after the administration of anti-IL-6 therapies, most commonly the IL-6R antagonist tocilizumab. ${ }^{114115117118}$

Case studies evaluating sarilumab, another anti-IL-6R therapy also surfaced, describing improvements in respiratory parameters ${ }^{96}$ and shortened time to clinical improvement in patients with lung consolidation ${ }^{119}$ with treatment. Interpretation of individual non-randomized studies is challenging, and cross-trial comparisons are difficult, because anti-IL-6 was initiated at different stages during the course of disease and a rapidly evolving standard of care meant that patients may or may not have received a heterogenous assortment of concomitant medications, including steroids and/or antivirals.

Multiple randomized trials have now evaluated IL-6directed therapies in the management of COVID-19. The phase III study investigating sarilumab was halted early for futility because it did not meet its primary and key secondary endpoints. ${ }^{120}$ However, topline results have become available from eight randomized trials evaluating tocilizumab: RCT-TCZ-COVID-19, CORIMUNO-TOCI-1, COVACTA, EMPACTA, BACC Bay TCZ, REMAP-CAP, RECOVERY, and TOCIBRAS. Characteristics and key outcomes from randomized trials are summarized in table 2.

Of the major randomized trials with available results, four met at least one primary endpoint for efficacy. The CORIMMUNO-TOCI-1 study, ${ }^{121}$ showed significant improvement for reducing the need for ventilation with tocilizumab treatment, despite the fact that the threshold for efficacy for improvement to a score of greater than 5 on the World Health Organization (WHO) Clinical Progression Scale ${ }^{122}$ was not met and there was no difference in 28-day mortality between the groups. In the EMPACTA trial, which investigated the safety and efficacy of tocilizumab in hospitalized, non-ventilated patients, with an emphasis on enrolling high-risk and racial and ethnic minority populations, tocilizumab was shown to reduce the rate of mechanical ventilation or death by day $28 .{ }^{123}$

Importantly, the two largest trials with data available both met primary and secondary efficacy endpoints. REMAP-CAP, which randomized patients to tocilizumab, sarilumab, or standard of care within 24 hours of receiving organ support found that tocilizumab and sarilumab were effective not only for the primary outcome of organ support-free days, but also all secondary outcomes, including 90-day survival, time to ICU and hospital discharge, and improvement according to WHO ordinal scale at day $14 .^{124}$ The RECOVERY trial similarly saw significant reductions in mortality rates as well as met key secondary endpoints such as reduced intubation rate and reduced requirement for dialysis with tocilizumab. ${ }^{125}$ 


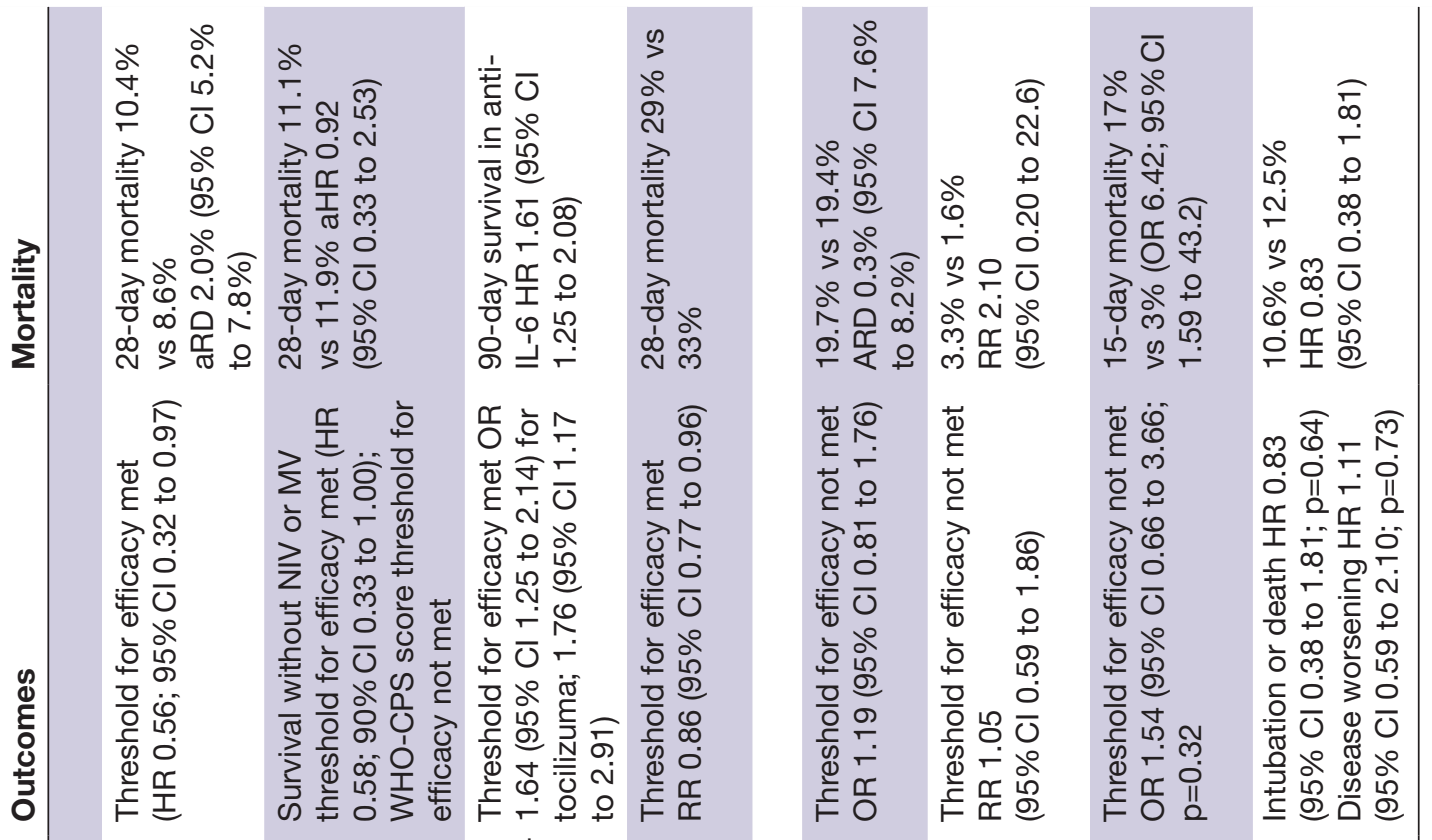

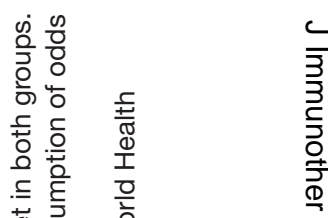

व
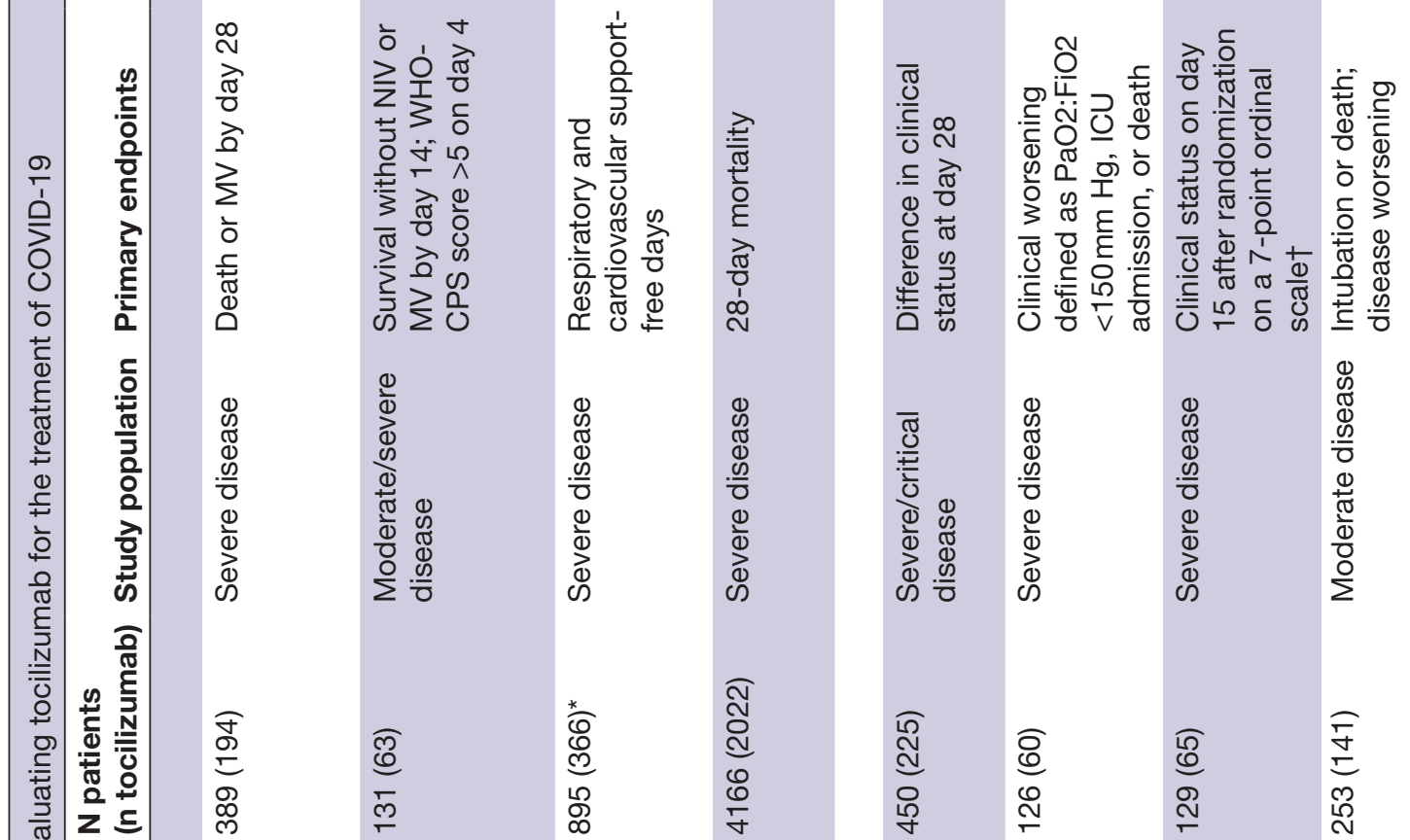

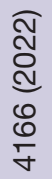
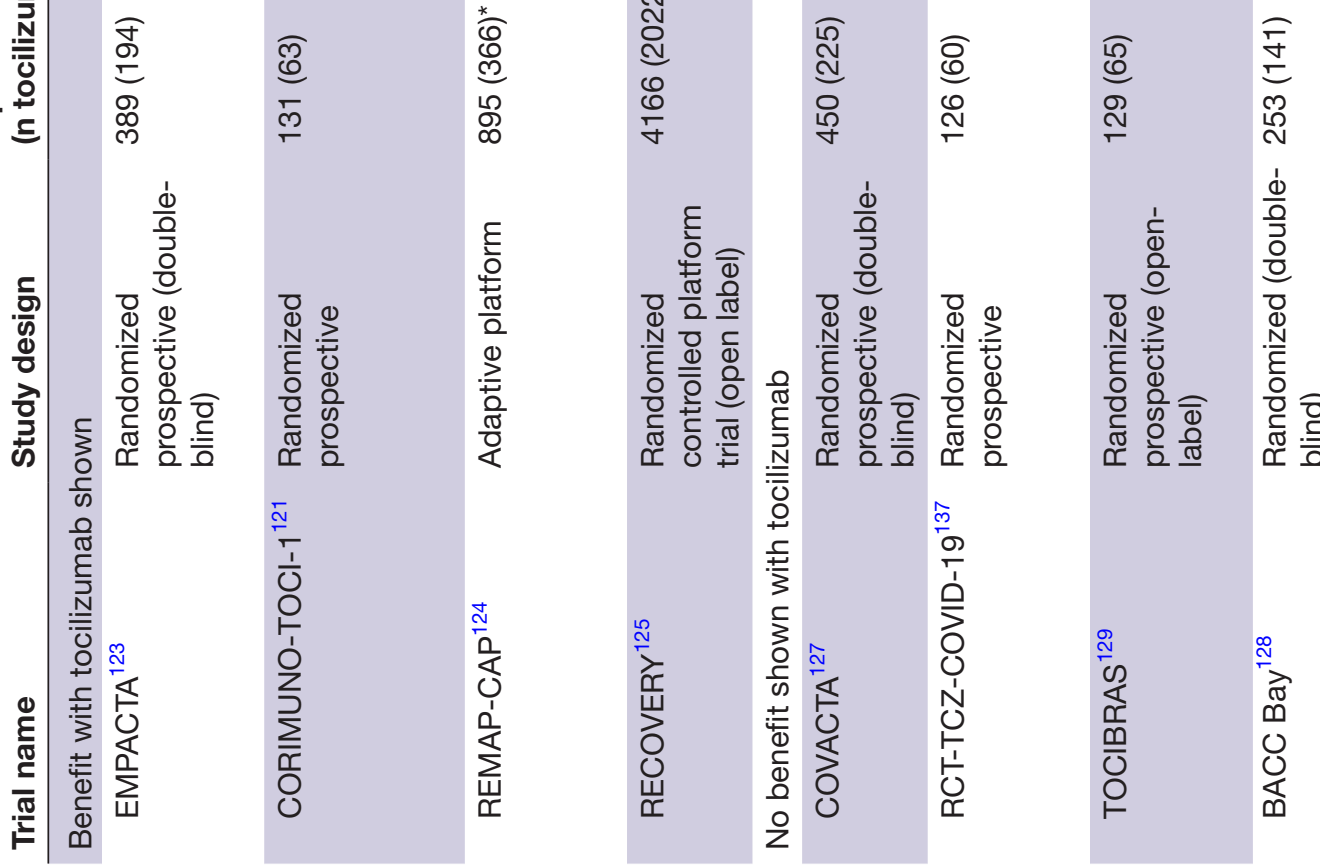

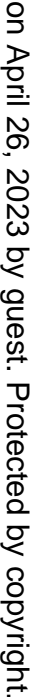


Although not randomized, the observational retrospective STOP-COVID study is also of note due to its large population size, as well as its stringent consistency with the timing of intervention-all enrolled patients received tocilizumab within 2 days of ICU admission. In the study, early tocilizumab treatment led to reduced risk of death across multiple sensitivity analyzes. ${ }^{126}$ In the study, the time to death HR with tocilizumab was 0.71 (95\% CI 0.56 to 0.92 ) and the 30 -day mortality risk difference (RD) was $9.6 \%$ (95\% CI $3.1 \%$ to $16.0 \%)$.

Four randomized studies did not meet their primary outcome endpoints; however, even in these trials, exploratory analyses suggest that anti-IL-6 may provide meaningful benefit for some patients. For example, although the COVACTA trial found no significant difference in clinical status at day 28 between study groups, the incidence of ICU transfer was $23.6 \%(30 / 127)$ in the tocilizumab arm and $40.6 \%(26 / 64)$ in the placebo arm (weighted difference, $-17.2 \% ; 95 \%$ CI $-31.3 \%$ to $-3.0 \%$; Cochran-Mantel-Haenszel nominal $\mathrm{p}=0.01$ ). In post hoc analysis of the patients not ventilated at randomization, more patients in the placebo group died, withdrew during hospitalization, were transferred to an ICU, or required invasive mechanical ventilation within 28 days of baseline (29.0\% (53 of 183 patients) in the tocilizumab arm and $42.2 \%$ (38 of 90) in the placebo) (HR 0.614; 95\% CI 0.40 to 0.94 ; nominal $\mathrm{p}=0.03) .{ }^{127}$ Additionally, although not statistically significant, numerically reduced duration of mechanical ventilation was observed with tocilizumab in the 19 patients who were intubated during the BACC Bay trial (median duration, 15.0 days in the tocilizumab group and 27.9 days in the placebo group).${ }^{128} \mathrm{~A}$ significant interaction was also found between treatment and required respiratory support in the TOCIVID-19 trial, such that the effect of tocilizumab on lethality was larger among patients not requiring mechanical ventilation (OR 0.37 (95\% CI 0.18 to 0.74 ) vs 0.50 (95\% CI 0.27 to 0.92$)$ ). ${ }^{116}$ TOCIBRAS, an open-label randomized trial conducted in Brazil was terminated early due to increased mortality at 15 days in the tocilizumab arm (8\% vs $20 \%$; odds ratio (OR) $1.54 ; 95 \%$ CI 0.66 to $3.66 ; \mathrm{p}=0.32$ ), though the difference was not statistically significant. However, despite not meeting mortality endpoints, patients treated with tocilizumab were found to have significantly reduced duration of hospital stay. ${ }^{129}$

Several meta-analyses have now evaluated the available cohort studies and randomized trials. ${ }^{130-132}$ Metaanalyses of randomized controlled trials found no effect on mortality (pooled risk ratio (RR) $1.09 ; 95 \%$ CI 0.80 to $1.49, \mathrm{I}^{2}=0 \%$ ), tocilizumab was associated reduced risk of mechanical ventilation (pooled RR $0.71 ; 95 \%$ CI 0.52 to $0.96 ; \mathrm{I}^{2}=0 \%$ ) and decreased risk of composite poor outcome (pooled RR 0.71; 95\% CI 0.56 to 0.89 ; $\left.\mathrm{I}^{2}=0 \%\right) .{ }^{132}$ By contrast, another analysis, which included both controlled trials and single-arm studies, found that mortality was slightly lower with tocilizumab compared with standard of care ( $\mathrm{RD}-0.06 ; 95 \% \mathrm{CI}-0.12$ to $\left.-0.01 ; \mathrm{p}=0.03 ; \mathrm{I}^{2}=80.9 \%\right)$, and the difference was more pronounced among the studies that included on only patients with severe disease (RD $-0.12 ; 95 \% \mathrm{CI}-0.18$ to $\left.-0.06 ; \mathrm{p}<0.01 ; \mathrm{I}^{2}=53.7 \%\right)$. The subgroup analysis for high quality studies was consistent, that is, lower mortality in TOC group (RD: $-0.07 ; 95 \%$ CI -0.13 to $-0.01 ; \mathrm{p}=0.02$; $\mathrm{I}^{2}=82.6 \%$ ). In that analysis, lower rates of mechanical ventilation with tocilizumab were only found in patients with severe COVID-19 (RD $-0.11 ; 95 \%$ CI -0.19 to -0.02 ; $\mathrm{p}=0.01 ; \mathrm{I}^{2}=74.0 \%$ for severe disease vs RD: $-0.04 ; 95 \% \mathrm{CI}$ -0.15 to $0.06 ; \mathrm{p}=0.44 ; \mathrm{I}^{2}=89.5 \%$; for the combined analysis). ${ }^{130}$ Reduced mortality with tocilizumab treatment was also demonstrated in a meta-analysis including nine high-quality small studies, with an RR of 0.27 (95\% CI 0.12 to 0.59 ) and $\mathrm{RD}$ of $12 \%$ (95\% CI $4.6 \%$ to $20 \%$ ) in favor of IL-6 blockade.

\section{Effect modulators: steroids, severity and timing?}

Cross-trial comparisons are complicated because the major studies evaluated tocilizumab in disparate patient populations at varying points in the COVID-19 disease course. Because hyperinflammation is a positive-feedback regulated response, intervention with anti-IL-6 either too early or too late in the disease course may negate any potential benefit. Furthermore, most patients admitted to the hospital for COVID-19 received multiple interventionsespecially at the beginning of the pandemic when options were limited, and efficacy data was sparse. Although remdesivir was initially shown to reduce the duration of hospitalization, ${ }^{133}$ updated WHO guidelines recommend against its use ${ }^{134}$ Other organizations, however, including the Infectious Disease Society of America (IDSA) recommend remdesivir over no antiviral treatment for hospitalized patients with severe COVID-19. ${ }^{135}$ Currently, the only intervention shown to significantly reduce mortality for severe COVID-19 is dexamethasone. ${ }^{136}$ Reflecting this ever-shifting landscape, patient in published trials may have received concomitant antivirals, corticosteroids, or other investigational agents along with IL-6-directed therapies. In addition to the potential confounding effects of comedications, the timing of administration and the patient selection criteria has varied between studies. The eligibility criteria for major studies of tocilizumab as well as the timing of administration of therapy and allowed concomitant medications is summarized in table 3 .

Disease severity may be an effect modulator. In one meta-analysis, the mortality benefits for tocilizumab was more pronounced among patients with severe disease (RD 12\%; $95 \%$ CI $18 \%$ to $6 \%$; $\mathrm{p}<0.01$ ) compared with the overall population (RD $6 \%$; $95 \%$ CI $12 \%$ to $1 \%$; $=0.03$ ), ${ }^{130}$ though the effects of steroids were not investigated. Another systematic review found that tocilizumab was associated with reduced mortality across disease severities (pooled adjusted RR 0.58 ; 95\% CI 0.51 to $0.66, \mathrm{I}^{2}=2.5 \%$ ), although the effect only held for cohort studies and not randomized trials. ${ }^{132}$ Supporting this, the REMAP-CAP trial recruited patients with severe disease, requiring organ support at the time of tocilizumab administration. In RECOVERY, 562 patients (14\%) were receiving 


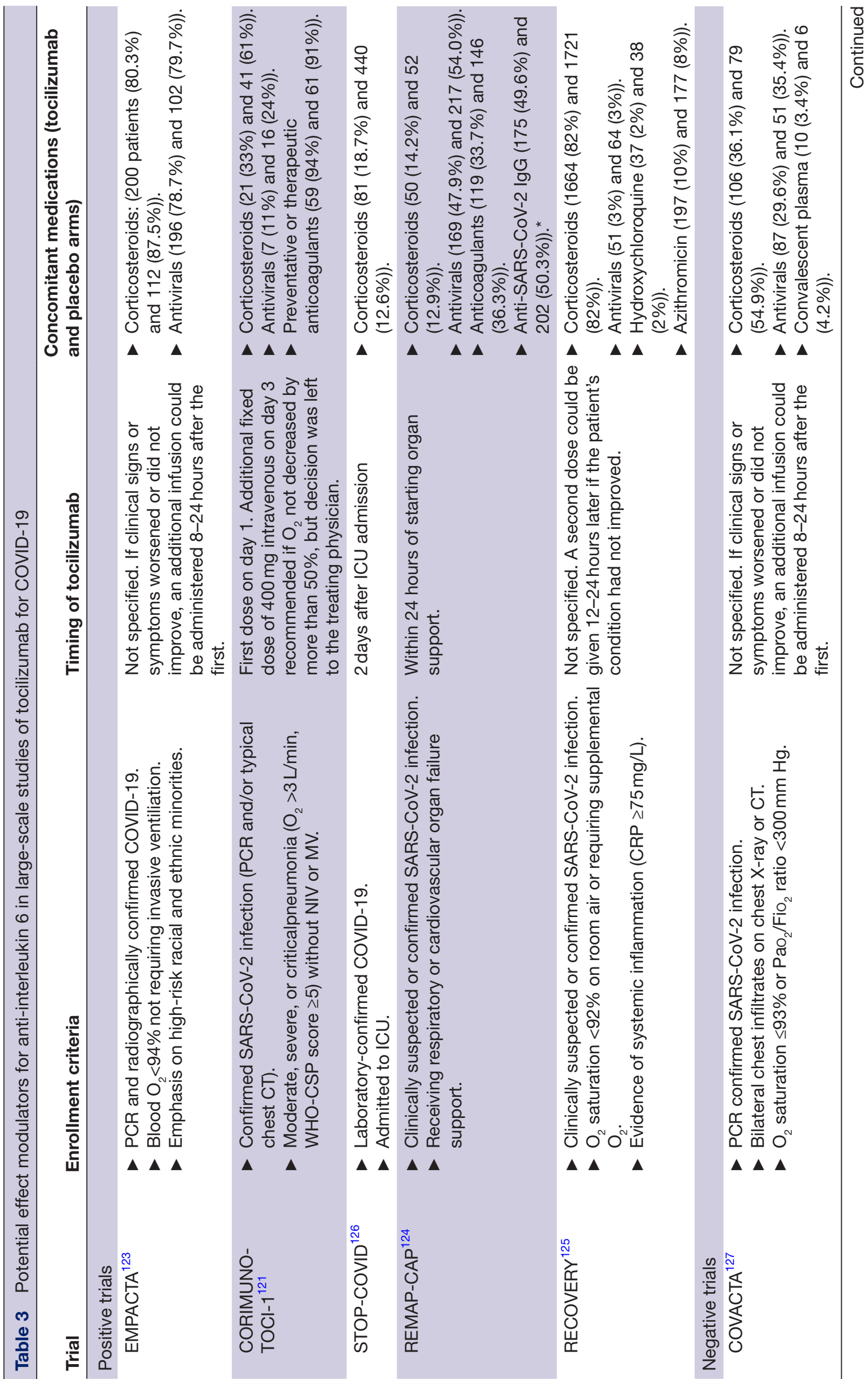




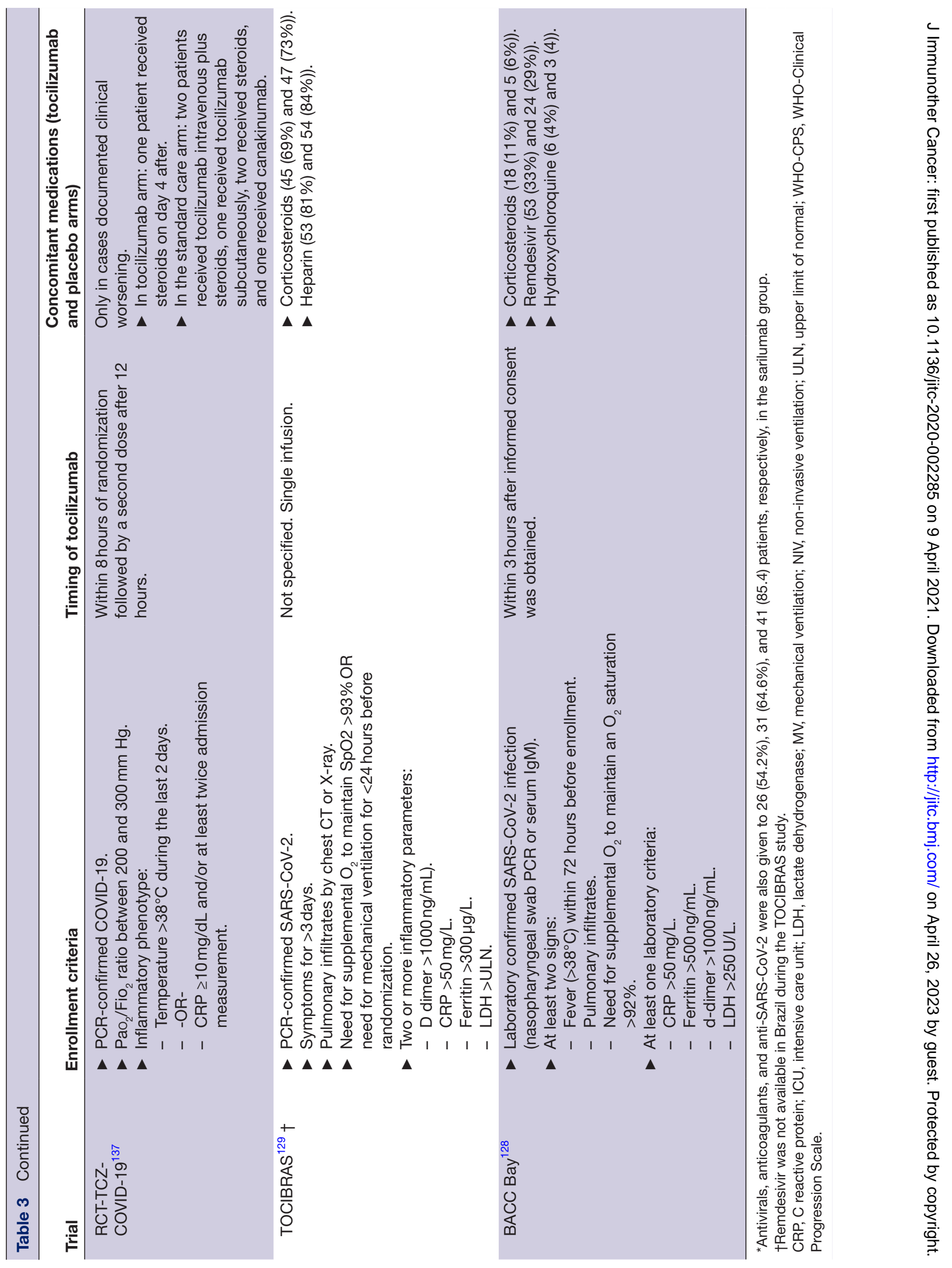


invasive mechanical ventilation at the time of hospitalization, while 1686 (41\%) required non-invasive respiratory support, and 1868 (45\%) were on supplemental oxygen. While more data are needed on tociliumab for moderate or mild COVID-19 (not requiring oxygen support), no benefit was seen in the BACC Bay trial that enrolled patients with minimal symptoms and only mildly elevated inflammatory parameters. ${ }^{128}$ The RCT-TCZ-COVID-19 trial also showed that early administration of tocilizumab in patients with $\mathrm{PaO}_{2} / \mathrm{FiO}_{2}$ between 200 and $300 \mathrm{~mm} \mathrm{Hg}$, and a mild inflammatory phenotype defined by fever or CRP $\geq 10 \mathrm{mg} / \mathrm{dL}$ did not reduce the risk of clinical worsening. ${ }^{137}$

Steroids are now recognized as an important component of effective interventions for COVID-19, although early reports paint conflicting pictures of the interplay between glucocorticoids and IL-6 antagonism. In the retrospective STOP-COVID trial, corticosteroid use was not shown to significantly affect mortality for tocilizumab treatment (HRs $0.71 ; 95 \%$ CI 0.53 to 0.96 with steroids vs 0.68 ; $95 \%$ CI 0.46 to 0.99 without; $\mathrm{p}=0.83$ ), although the proportion of patients who received steroids in that trial was low. ${ }^{126}$ A subsequent meta-analysis found that glucocorticoid use was associated with smaller effects of tocilizumab on mortality with an RD of $9.1 \%$ (95\% CI $2.8 \%$ to $15 \%$ ) compared with $31 \%$ (95\% CI $15 \%$ to $47 \%$ ). Importantly, however, no difference in mortality was seen between the tocilizumab and control groups for studies that used steroids without antivirals. ${ }^{131}$ Corticosteroids use was also associated with a lower OR for death both at 14 and 30 days (OR 0.36 (95\% CI 0.21 to 0.62 ) and OR 0.62 (95\% CI 0.40 to 0.95$)$ ) in the TOCIVID-19 study. ${ }^{116}$ In the TOCIBRAS trial, where corticosteroids use was evenly balanced between treatment groups, yet remdesivir was not available in Brazil, increased mortality was seen in the tocilizumab arm. The interpretation of these data is challenging because the sample size is small and the majority of patients were treated at a single center. ${ }^{129}$ By contrast, results from REMAP-CAP and RECOVERY support the benefits of combining IL-6 modulation with corticosteroids for COVID-19. At the time of randomization, $82 \%$ of patients in RECOVERY and $93 \%$ of patients in REMAP-CAP were receiving steroids. In both studies, the combination of anti-IL-6 and corticosteroids led to greater magnitude of benefit than any single intervention alone. $^{124} 125$

The historically controlled prospective CHIC trial found that a 5-day course of high-dose methylprednisolone followed by dose-reduction for the steroid and tocilizumab administration led to a $79 \%$ higher likelihood of reaching $\geq 2$ stages of improvement on the 7 -item WHOendorsed disease severity scale (HR $1.8 ; 95 \%$ CI 1.2 to 2.7 ) as well as $65 \%$ less mortality (HR 0.35 ; $95 \%$ CI 0.19 to 0.65 ), and $71 \%$ less need for invasive mechanical ventilation (HR $0.29 ; 95 \%$ CI 0.14 to 0.65$).{ }^{138}$ The CHIC study was notable because it incorporated a lab parameterbased selection to enroll only patients experiencing a COVID-19-related cytokine storm (defined as rapid respiratory deterioration plus elevation in at least two out of three biomarkers: CRP $>100 \mathrm{mg} / \mathrm{L}$, ferritin $>900 \mu \mathrm{g} / \mathrm{L}$ or d-dimer $>1500 \mu \mathrm{g} / \mathrm{L}$ ).

Another retrospective study found that timing of administration of anti-IL-6 had important effects on outcomes among patients with severe COVID-19 (defined as a $\mathrm{SpO}_{2} / \mathrm{FiO}_{2}<325$ with bilateral pneumonia and a clinical diagnosis of infection with SARS-CoV-2) who were treated with both tocilizumab and steroids. In the early therapy subgroup who received tocilizumab after 24 hours of admission and before $\mathrm{SpO}_{2} / \mathrm{FiO}_{2}$ decreased to $\leq 250,6.25 \%$ required ICU admission or died in hospital. By contrast, mortality or ICU admission occurred in $45.3 \%$ of patients who received tocilizumab and steroids when $\mathrm{SpO}_{2} / \mathrm{FiO}_{2}$ was $<250$ (6.25\% vs $34.9 \%$, $\mathrm{p}<0.01) .{ }^{139}$

Other effect modulators that may underlie inter- and intra-trial heterogeneity include geography, patient ethnicity, comorbidities, and the microbiota. Marked regional differences in mortality due to COVID-19 have been observed, ${ }^{140}$ with proposed mechanisms involving differences in vitamin D levels by latitude, geographic and dietary influences on the gut microbiome, and others. Altered composition of the bacterial and fungal microbiota have been correlated with disease severity in COVID-19. ${ }^{141-143}$ Bacterially produced butyrate and other short-chain fatty acids have been shown to modulate $\mathrm{CD}^{+} \mathrm{T}$ cell effector functions as well as IL-6 production by monocytes, ${ }^{144} 145$ a potential mechanism linking dysbiosis to COVID-19 disease severity. Although not currently actionable, interactions between the gut microbiota and SARS-CoV-2 pathogenesis may contribute to the clinical course of COVID-19. Future studies may identify microbiota-targeted perturbations or patient selection strategies to enhance the efficacy of anti-IL-6.

It has also become apparent that racial and ethnic minorities experience disproportionately high mortality rates from COVID-19 in the USA and the UK. ${ }^{3146}$ The confounding effects of differential access to healthcare, comorbidities, and demographics complicates the interpretation of these results, however. ${ }^{146}$ Despite being multicenter, international trials, patients in REMAP-CAP and RECOVERY were majority white (roughly $70 \%$ ), whereas TOCIBRAS was conducted in Brazil. Of note, EMPACTA trial, which recruited patients with an emphasis on inclusion of racial minorities met its primary efficacy endpoint of reduced 28-day mortality with tocililzumab in a population that was $56.0 \%$ Hispanic or Latino, $14.9 \%$ Black, $12.7 \%$ American Indian or Alaska Native, $12.7 \%$ nonHispanic White, and $3.7 \%$ were of other or unknown race or ethnic group. ${ }^{123}$ Recruiting diverse patient populations should remain a priority for ongoing and future trials, both to identify any potential biological differences in response to therapy among different groups, as well as to improve access to care. 


\section{DEFINING THE WINDOW OF OPPORTUNITY}

Taken together, the results of randomized trials and metaanalyses clearly demonstrate that anti-IL-6 therapies are not universally effective for COVID-19, however, cytokine modulation offers definitive benefit for some patients, if administered at the appropriate time after symptom onset. Additionally, the optimal use of tocilizumab is likely in combination with glucocorticoids. Updated guidelines from IDSA reflect this evolving understanding of the standard of care, now suggesting the addition of anti-IL-6 to standard care (ie, steroids) in hospitalized patients with severe disease and elevated levels of inflammatory markers. ${ }^{147}$ In patients where the inflammatory cascade has advanced beyond a "point of no return" modulation of IL-6 is unlikely to provide benefit-exemplified by the futility of tocilizumab administration in the COVACTA trial, ${ }^{127}$ which included patients with critical disease. Conversely, IL-6 blockade too early in the disease course is also ill-advised. Because IL-6 a master regulator of inflammation and immunity, anti-cytokine therapy during the initial stages of infection may hinder the development of robust anti-viral $\mathrm{T}$ cell responses. ${ }^{101} 148$

Optimal incorporation of anti-IL-6 into the antiCOVID-19 arsenal will require defined criteria for the patients who may benefit. Parameters for patient selection should be readily available, and biologically informed. Trials to date have used CRP as marker for patient selection, and prespecified analysis in REMAP-CAP demonstrated the greatest magnitude of benefit with anti-IL-6 in the highest tercile for pretreatment CRP. Based on the validated definitions of COVID-19 hyperinflammation, ${ }^{77} 78$ and evidence from the large anti-IL-6 trials, a threshold of CRP $>75 \mathrm{mg} / \mathrm{L}$ likely may be used to identify patients for anti-IL-6 therapy. This threshold is the same value used for eligibility in the RECOVERY trial. ${ }^{125}$ Notably, this value for CRP is higher than the cut-off for inflammatory markers used in the BACC Bay trial, which showed no benefit for tocilizumab administration in moderate disease. ${ }^{128}$

\section{FUTURE DIRECTIONS AND OTHER ANTI-INFLAMMATORY STRATEGIES}

Anti-IL-6 therapies remain an ongoing area of investigation, both alone and in combination with other interventions such as steroids and antivirals. The phase III REMDACTA trial (NCT04409262), evaluating tocilizumab with remdesevir for severe COVID-19 pneumonia, is ongoing. Additional trials are studying the effects of varying the dose or route of administration of anti-IL-6, and at least one case report has shown benefit with subcutaneous tocilizumab. ${ }^{149}$ Future studies of anti-IL-6 therapies should include detailed biomarker analyses at baseline and on-treatment to definitively establish indicators of response to therapy as well as tools for patient selection.

Additionally, future studies will be needed to rule out the potential for harm with tocilizumab treatment, specifically the possibility of further immunosuppression leading to secondary infection. The FDA label for tocilizumab includes a black boxed warning that serious infections leading to hospitalization or death including tuberculosis, bacterial, invasive fungal, viral, and other opportunistic infections have occurred in patients on treatment for rheumatoid arthritis. Some retrospective studies have reported increased secondary infections in patients with COVID-19 treated with tocilizumab. ${ }^{150-152}$ No such increased risk was seen in several single-center randomized controlled trials, however. ${ }^{116} 121128137$ Similarly, the RECOVERY trial reported no significant increase in the rate of secondary infections with IL- 6 modulation, ${ }^{125}$ and only one secondary bacterial infection was observed in the tocilizumab group in the REMAP-CAP study. ${ }^{124}$ Notably, experience from CAR $\mathrm{T}$ cell therapy indicates that tocilizumab administration for CRS is not associated with increased infection risk. Because the "single shot" tocilizumab dosing used for COVID-19 is more akin to CRS management than the long-term immunosuppression required to treat rheumatoid arthritis, the risk for secondary infections may be reduced. Future metaanalyses, however, will be necessary to determine if the single or double doses of tocilizumab given in the context of COVID-19 is associated with a significant risk signal for secondary infections.

Several other anti-inflammatory and immunemodulatory strategies have been proposed to calm the cytokine storm in COVID-19. ${ }^{153}$ Targeting the monocytedriven inflammatory pathology through the administration of anti-GM-CSF antibodies is an active area of investigation. ${ }^{63154}$ Modulation of IL-1 $\beta$ with anakinra has demonstrated improved survival in cohort-controlled studies, ${ }^{155} 156$ although it remains to be seen if the results are recapitulated in randomized trials. Targeting downstream components of the IL- 6 signaling cascade is also being studied, with several janus kinase inhibitors being repurposed for COVID-19. ${ }^{153}$ Of note, baricitinib, which was identified through artificial intelligence as a potential treatment, reportedly led to prompt resolution of respiratory function and improvement radiological findings when used as a rescue therapy for a patient with disease that did not respond to sarilumab or antivirals. ${ }^{157}$ Baricitinib has been evaluated in the phase III ACTT-2 study (NCT04401579), which enrolled 1034 hospitalized patients and found that JAK inhibition plus remdesivir was superior to the antiviral alone in reducing recovery time and accelerating improvement in clinical status. ${ }^{158}$ Several small-scale studies are ongoing, and active phase III trials evaluating baricitinib and anakinra for COVID-19 are summarized in table 4 .

\section{CONCLUSION}

The current standard of care for severe COVID-19 is dexamethasone, which has conclusively been shown to reduce mortality in patients requiring supplemental oxygen. ${ }^{136}$ There is currently no generally accepted recommended 


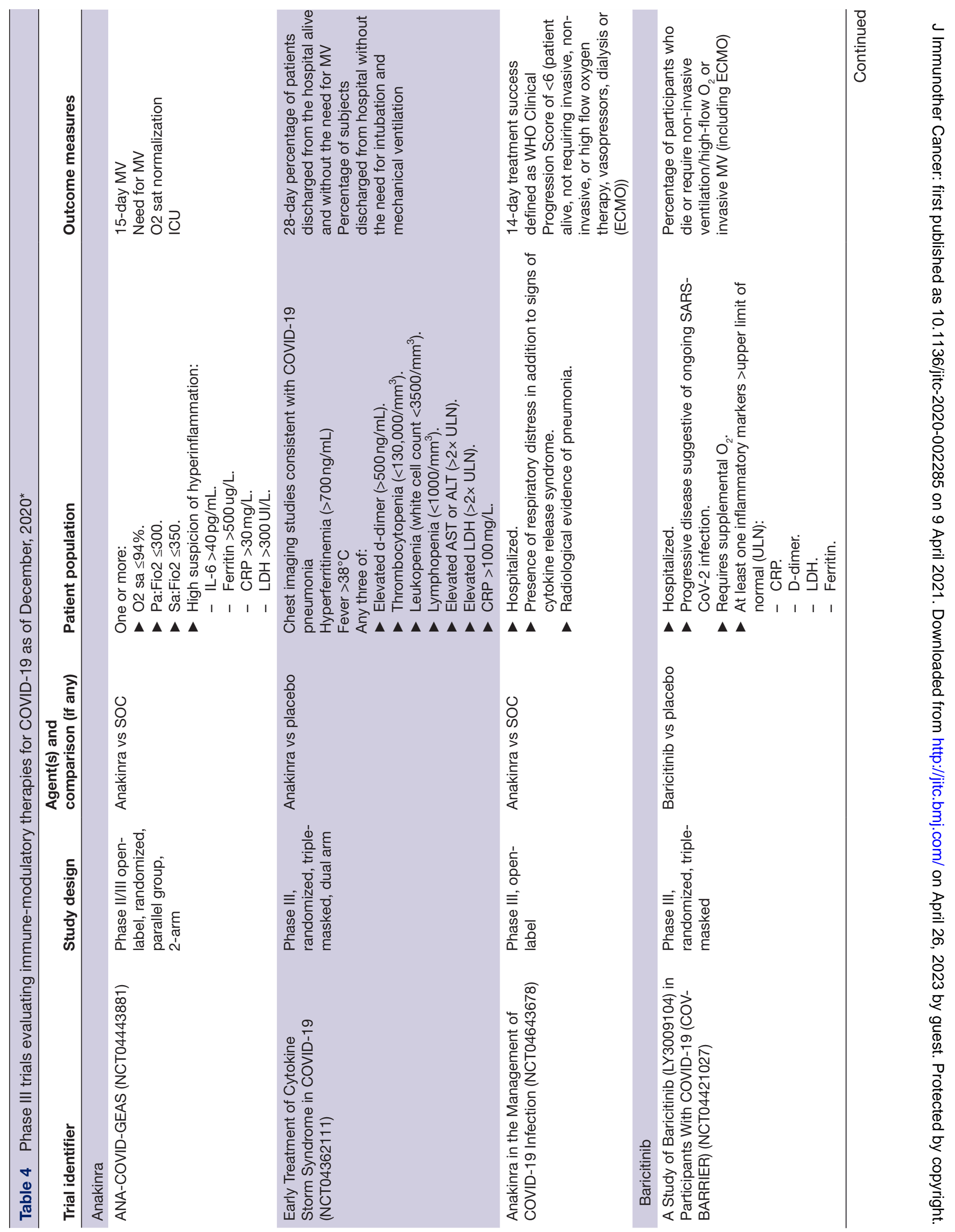




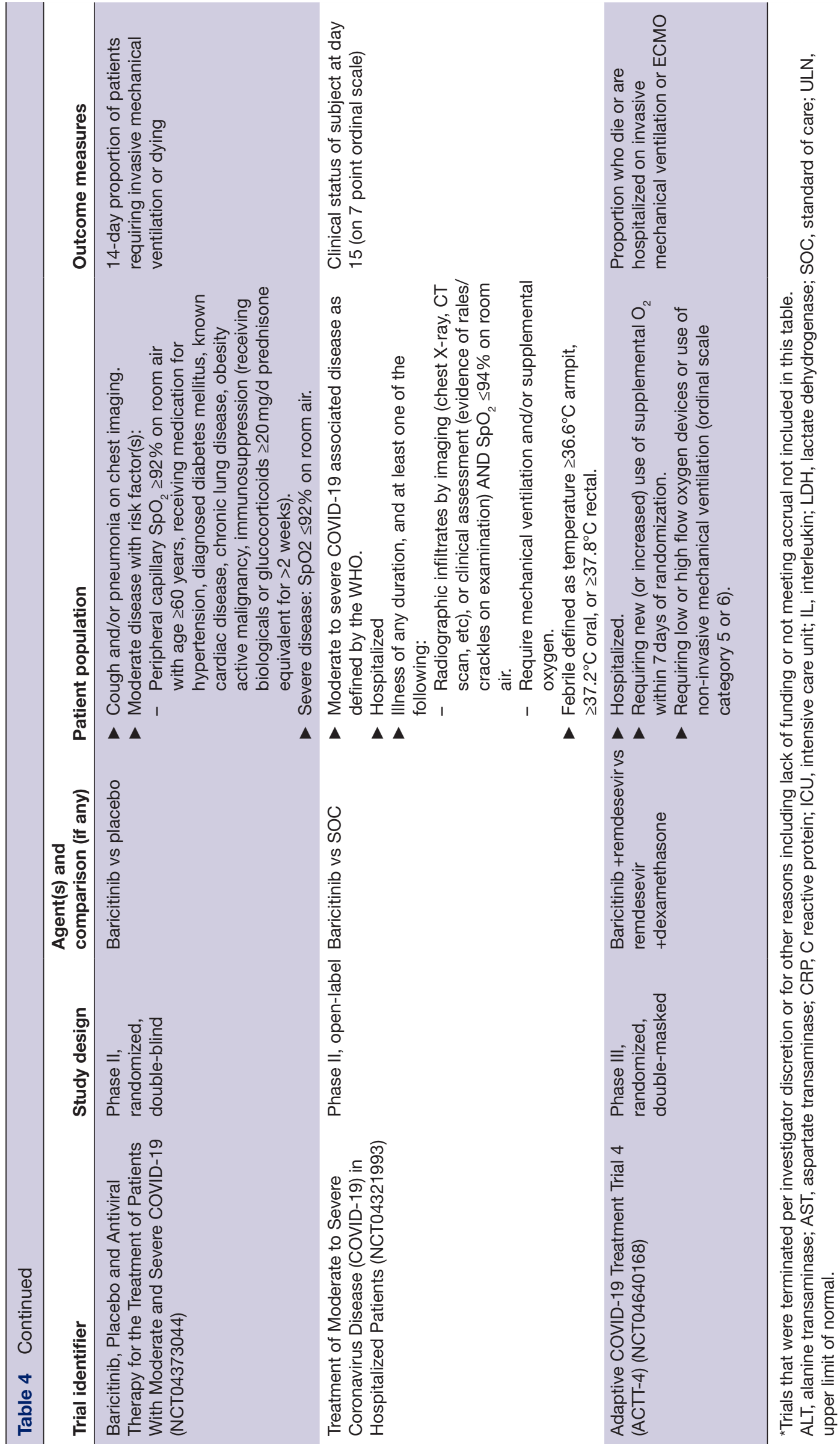


therapy for patients with mild or moderate disease, yet increasing evidence suggests that adding anti-IL-6 to corticosteroids for hypoxic patients may reduce mortality and shorten times in hospital. Although vaccine rollout is ramping up, the healthcare system has been strained beyond capacity, and interventions that reduce the burden on hospital resources are still desperately needed. In future trials, it will be important to evaluate endpoints beyond mortality, such as need for ICU care, duration of hospital stay, or need for ventilation, so that therapies that alleviate the demands on the healthcare system may be identified. Additionally, trials that specifically recruit participants from historically under-represented populations, such as was done in the EMPACTA study, ${ }^{123}$ will be important to not only provide equitable access to therapy but also potentially identify discrepancies in response to treatment among demographics.

In all likelihood, the optimal treatment approach for COVID-19 will be context-dependent and involve a combination of several different therapies. Establishing clinical benefit in such a complex landscape-a heterogeneous disease treated with a combination of therapies-may be challenging. For this reason, the immuno-oncology community may be uniquely poised to support our colleagues in infectious disease and intensive care. Cancer clinical trialists have extensive experience in the design and execution of adaptive, biomarker-selected studies that involve complex combination regimens. Additionally, immunotherapy practitioners may contribute expertize in assay development and sample handling considerations for the delicate and difficult immunological profiling experiments that will be necessary to gain additional insight into the pathology of COVID-19. As the world responds to COVID-19, interdisciplinary collaboration will be essential, and the lessons learned from this pandemic may provide crucial insight, not only in the next infectious outbreak, but in understanding the basic mechanisms of inflammation and immunity.

\section{Twitter Paolo Antonio Ascierto @PAscierto}

Contributors All the authors contributed to the conception, content, writing and revisions, and have read and approved the submitted manuscript.

Funding The authors have not declared a specific grant for this research from any funding agency in the public, commercial or not-for-profit sectors.

Competing interests PAA has/had a consultant/advisory role for Bristol Myers Squibb, Roche-Genentech, Merck Sharp \& Dohme, Novartis, Array, Merck Serono, Pierre-Fabre, Incyte, Medimmune, AstraZeneca, Syndax, Sun Pharma, Sanofi, Idera, Ultimovacs, Sandoz, Immunocore, 4SC, Alkermes, Italfarmaco, Nektar, BoehringerIngelheim, Eisai, Regeneron, Daiichi Sankyo, Pfizer, Oncosec, Nouscom, Takis, Lunaphore. He also received research funding from Bristol Myers Squibb, RocheGenentech, Array, Sanofi and travel support from MSD. All the other authors have no conflict of interest to declare.

\section{Patient consent for publication Not required}

Provenance and peer review Commissioned; externally peer reviewed.

Open access This is an open access article distributed in accordance with the Creative Commons Attribution Non Commercial (CC BY-NC 4.0) license, which permits others to distribute, remix, adapt, build upon this work non-commercially, and license their derivative works on different terms, provided the original work is properly cited, appropriate credit is given, any changes made indicated, and the use is non-commercial. See http://creativecommons.org/licenses/by-nc/4.0/.
ORCID iD

Paolo Antonio Ascierto http://orcid.org/0000-0002-8322-475X

\section{REFERENCES}

1 JohnsHopkins. Mortality analyses - Johns Hopkins Coronavirus resource center: JohnsHopkins, 2020. Available: https:// coronavirus.jhu.edu/data/mortality

2 Petersen E, Koopmans M, Go U, et al. Comparing SARS-CoV-2 with SARS-CoV and influenza pandemics. Lancet Infect Dis 2020;20:e238-44.

3 Rossen LM BA, Ahmad FB, Sutton P, et al. Excess deaths associated with COVID-19, by age and race and ethnicity - United States, January 26-October 3, 2020 | MMWR. Morb Mortal Wkly Rep;2020:1522-7.

4 Richards M, Anderson M, Carter P, et al. The impact of the COVID-19 pandemic on cancer care. Nature Cancer 2020;1:565-7.

5 Brar G, Pinheiro LC, Shusterman M, et al. COVID-19 severity and outcomes in patients with cancer: a matched cohort study. J Clin Oncol 2020;38:3914-24.

6 Giannakoulis VG, Papoutsi E, Siempos II. Effect of cancer on clinical outcomes of patients with COVID-19: a meta-analysis of patient data. JCO Glob Oncol 2020;6:799-808.

7 Garassino MC, Whisenant JG, Huang L-C, et al. COVID-19 in patients with thoracic malignancies (TERAVOLT): first results of an international, registry-based, cohort study. Lancet Oncol 2020;21:914-22.

8 Chen G, Wu D, Guo W, et al. Clinical and immunological features of severe and moderate coronavirus disease 2019. Journal of Clinical Investigation 2020;130:2620-9.

9 Huang C, Wang Y, Li X, et al. Clinical features of patients infected with 2019 novel coronavirus in Wuhan, China. The Lancet 2020;395:497-506.

10 Wang Z, Yang B, Li Q, et al. Clinical features of 69 cases with coronavirus disease 2019 in Wuhan, China. Clin Infect Dis 2020;71:769-77.

11 Grasselli G, Zangrillo A, Zanella A, et al. Baseline characteristics and outcomes of 1591 patients infected with SARS-CoV-2 admitted to ICUs of the Lombardy region, Italy. JAMA 2020;323:1574-81.

12 Guan W-jie, Liang W-hua, Zhao Y, et al. Comorbidity and its impact on 1590 patients with COVID-19 in China: a nationwide analysis. Eur Respir J 2020;55:2000547.

13 Kalligeros M, Shehadeh F, Mylona EK, et al. Association of obesity with disease severity among patients with coronavirus disease 2019. Obesity 2020;28:1200-4.

14 Onder G, Rezza G, Brusaferro S. Case-Fatality rate and characteristics of patients dying in relation to COVID-19 in Italy. JAMA 2020.

15 Simonnet A, Chetboun M, Poissy J, et al. High prevalence of obesity in severe acute respiratory syndrome coronavirus-2 (SARS-CoV-2) requiring invasive mechanical ventilation. Obesity 2020;28:1195-9.

16 Zhou F, Yu T, Du R, et al. Clinical course and risk factors for mortality of adult inpatients with COVID-19 in Wuhan, China: a retrospective cohort study. The Lancet 2020;395:1054-62.

17 Bastard P, Rosen LB, Zhang Q, et al. Autoantibodies against type I IFNs in patients with life-threatening COVID-19. Science 2020;370:eabd4585.

18 Zhang Q, Bastard P, Liu Z, et al. Inborn errors of type I IFN immunity in patients with life-threatening COVID-19. Science 2020;370:eabd4570.

19 Liu Y, Du X, Chen J. Neutrophil-To-Lymphocyte ratio as an independent risk factor for mortality in hospitalized patients with COVID-19. J Infect 2020;81:e6-12.

20 Yang A-P, Liu J-P, Tao W-Q, et al. The diagnostic and predictive role of NLR, d-NLR and PLR in COVID-19 patients. Int Immunopharmacol 2020;84:106504.

21 Boyiadzis M, Bishop MR, Abonour R, et al. The Society for immunotherapy of cancer consensus statement on immunotherapy for the treatment of hematologic malignancies: multiple myeloma, lymphoma, and acute leukemia. J Immunother Cancer 2016;4:90.

22 Herold T, Jurinovic V, Arnreich C, et al. Elevated levels of IL-6 and CRP predict the need for mechanical ventilation in COVID-19. J Allergy and Immunol 2020;146:128-36.

23 Laguna-Goya R, Utrero-Rico A, Talayero P, et al. IL-6-based mortality risk model for hospitalized patients with COVID-19. Journal of Allergy and Clinical Immunology 2020;146:799-807.

24 Fajgenbaum DC, June CH. Cytokine storm. N Engl J Med 2020;383:2255-73. 
25 McGonagle D, Sharif K, O'Regan A, et al. The role of cytokines including interleukin-6 in COVID-19 induced pneumonia and macrophage activation syndrome-like disease. Autoimmun Rev 2020;19:102537.

26 Opoka-Winiarska V, Grywalska E, Roliński J. Could hemophagocytic lymphohistiocytosis be the core issue of severe COVID-19 cases? BMC Med 2020;18:214.

27 Pedersen SF, Ho Y-C. SARS-CoV-2: a storm is raging. Journal of Clinical Investigation 2020;130:2202-5.

$28 \mathrm{Xu} \mathrm{X}$, Han M, Li T, et al. Effective treatment of severe COVID-19 patients with tocilizumab. Proc Natl Acad Sci U S A 2020;117:10970-5.

29 Zhang W, Zhao Y, Zhang F, et al. The use of anti-inflammatory drugs in the treatment of people with severe coronavirus disease 2019 (COVID-19): the perspectives of clinical immunologists from China. Clinical Immunology 2020;214:108393.

30 Ascierto PA ea. SITC Statement on anti-IL-6/IL-6R for COVID-19 - Society for Immunotherapy of Cancer (SITC), 2020. Available: https://www.sitcancer.org/research/covid-19-resources/il-6editorial

31 Lai C-C, Liu YH, Wang C-Y, et al. Asymptomatic carrier state, acute respiratory disease, and pneumonia due to severe acute respiratory syndrome coronavirus 2 (SARS-CoV-2): facts and myths. $J$ Microbiol Immunol Infect 2020;53:404-12.

32 Mo P, Xing Y, Xiao Y, et al. Clinical characteristics of refractory COVID-19 pneumonia in Wuhan, China. Clin Infect Dis 2020

33 Richardson S, Hirsch JS, Narasimhan M, et al. Presenting characteristics, comorbidities, and outcomes among 5700 patients hospitalized with COVID-19 in the new York City area. JAMA 2020;323:2052.

34 Rodriguez-Morales AJ, Cardona-Ospina JA, Gutiérrez-Ocampo $\mathrm{E}$, et al. Clinical, laboratory and imaging features of COVID-19: a systematic review and meta-analysis. Travel Med Infect Dis 2020;34:101623.

35 del Rio C, Collins LF, Malani P. Long-Term health consequences of COVID-19. JAMA 2020;324:1723-4.

36 Rehman S, Majeed T, Ansari MA, et al. Syndrome resembling Kawasaki disease in COVID-19 asymptomatic children. $J$ Infect Public Health 2020;13:1830-2.

37 Verdoni L, Mazza A, Gervasoni A, et al. An outbreak of severe Kawasaki-like disease at the Italian epicentre of the SARSCoV-2 epidemic: an observational cohort study. The Lancet 2020;395:1771-8

38 Du Y, Tu L, Zhu P, et al. Clinical features of 85 fatal cases of COVID-19 from Wuhan. A retrospective observational study. Am J Respir Crit Care Med 2020;201:1372-9.

39 Agarwal WKP. Chest imaging appearance of COVID-19 infection, 2020. Available: https://doiorg/101148/ryct2020200028

40 Gattinoni L, Chiumello D, Caironi P, et al. COVID-19 pneumonia: different respiratory treatments for different phenotypes? Intensive Care Med 2020;46:1099-102.

41 Robba C, Battaglini D, Ball L, et al. Distinct phenotypes require distinct respiratory management strategies in severe COVID-19. Respir Physiol Neurobiol 2020;279:103455.

42 Jin C, Tian C, Wang Y, et al. A pattern categorization of CT findings to predict outcome of COVID-19 pneumonia. Frontiers in Public Health 2020;8:567672.

43 Tian S, Hu W, Niu L, et al. Pulmonary pathology of early-phase 2019 novel coronavirus (COVID-19) pneumonia in two patients with lung cancer. J Thorac Oncol 2020;15:700-4

$44 \mathrm{Xu}$ Z, Shi L, Wang Y, et al. Pathological findings of COVID-19 associated with acute respiratory distress syndrome. The Lancet Respiratory Medicine 2020;8:420-2.

45 Rapkiewicz AV, Mai X, Carsons SE, et al. Megakaryocytes and platelet-fibrin thrombi characterize multi-organ thrombosis at autopsy in COVID-19: a case series. EClinicalMedicine 2020;24:100434

46 Magro C, Mulvey JJ, Berlin D, et al. Complement associated microvascular injury and thrombosis in the pathogenesis of severe COVID-19 infection: a report of five cases. Translational Research 2020;220:1-13

47 Nicholls JM, Poon LLM, Lee KC, et al. Lung pathology of fatal severe acute respiratory syndrome. The Lancet 2003;361:1773-8.

48 Bryce C, Grimes Z, Pujadas E. Pathophysiology of SARS-CoV-2: targeting of endothelial cells renders a complex disease with thrombotic microangiopathy and aberrant immune response. The Mount Sinai COVID-19 autopsy experience. medRxiv 2020.

49 Diao B, Wang C, Tan Y, et al. Reduction and functional exhaustion of T cells in patients with coronavirus disease 2019 (COVID-19). Front Immunol 2020;11.
50 Hoffmann M, Kleine-Weber H, Schroeder S, et al. SARS-CoV-2 cell entry depends on ACE2 and TMPRSS2 and is blocked by a clinically proven protease inhibitor. Cell 2020;181:271-80.

51 Shang J, Wan Y, Luo C, et al. Cell entry mechanisms of SARSCoV-2. Proc Natl Acad Sci U S A 2020;117:11727-34.

52 Tang X, Yang M, Duan Z. Transferrin receptor is another receptor for SARS-CoV-2 entry. bioRxiv 2020.

53 Ou X, Liu Y, Lei X, et al. Characterization of spike glycoprotein of SARS-CoV-2 on virus entry and its immune cross-reactivity with SARS-CoV. Nat Commun 2020;11:1-12.

54 Harmer D, Gilbert M, Borman R, et al. Quantitative mRNA expression profiling of ACE 2, a novel homologue of angiotensin converting enzyme. FEBS Lett 2002;532:107-10.

55 Hamming I, Timens W, Bulthuis MLC, et al. Tissue distribution of ACE2 protein, the functional receptor for SARS coronavirus. A first step in understanding SARS pathogenesis. J Pathol 2004;203:631-7.

56 Young MJ, Clyne CD, Chapman KE. Endocrine aspects of ACE2 regulation: RaaS, steroid hormones and SARS-CoV-2. Journal of Endocrinology 2020;247:R45-62.

57 Tikellis C, Thomas MC. Angiotensin-converting enzyme 2 (ACE2) is a key modulator of the renin angiotensin system in health and disease. Int J Pept 2012;2012:1-8.

58 Bradley BT, Maioli H, Johnston R, et al. Histopathology and ultrastructural findings of fatal COVID-19 infections in Washington state: a case series. The Lancet 2020;396:320-32.

59 Hou YJ, Okuda K, Edwards CE, et al. SARS-CoV-2 reverse genetics reveals a variable infection gradient in the respiratory tract. Cell 2020;182:429-46

60 Schaefer I-M, Padera RF, Solomon IH, et al. In situ detection of SARS-CoV-2 in lungs and airways of patients with COVID-19. Mod Pathol 2020;33:2104-14.

61 Bussani R, Schneider E, Zentilin L, et al. Persistence of viral RNA pneumocyte syncytia and thrombosis are hallmarks of advanced COVID-19 pathology. EBioMedicine 2020;61:103104.

62 Lindsley AW, Schwartz JT, Rothenberg ME. Eosinophil responses during COVID-19 infections and coronavirus vaccination. J Allergy Clin Immunol 2020;146:1-7.

63 Gómez-Rial J, Rivero-Calle I, Salas A, et al. Role of Monocytes/ Macrophages in Covid-19 Pathogenesis: Implications for Therapy]l\&gt. Infect Drug Resist 2020;13:2485-93.

64 Zhang H, Zhou P, Wei Y, et al. Histopathologic changes and SARSCoV-2 immunostaining in the lung of a patient with COVID-19. Ann Intern Med 2020;172:629-32.

65 Lee DW, Santomasso BD, Locke FL, et al. ASTCT consensus grading for cytokine release syndrome and neurologic toxicity associated with immune effector cells. Biology of Blood and Marrow Transplantation 2019;25:625-38

66 Brudno JN, Kochenderfer JN. Toxicities of chimeric antigen receptor T cells: recognition and management. Blood 2016;127:3321-30.

67 Brudno JN, Kochenderfer JN. Recent advances in car T-cell toxicity: mechanisms, manifestations and management. Blood Rev 2019;34:45-55

68 Frey N, Porter D. Cytokine release syndrome with chimeric antigen receptor T cell therapy. Biology of Blood and Marrow Transplantation 2019;25:e123-7.

69 Neelapu SS. Managing the toxicities of car T-cell therapy. Hematol Oncol 2019;37:48-52.

70 Morimoto A, Nakazawa Y, Ishii E. Hemophagocytic lymphohistiocytosis: pathogenesis, diagnosis, and management. Pediatrics International 2016;58:817-25.

71 Fujiwara F, Hibi S, Imashuku S. Hypercytokinemia in hemophagocytic syndrome. J Pediatr Hematol Oncol 1993;15:92-8.

72 La Rosée P. Treatment of hemophagocytic lymphohistiocytosis in adults. Hematology 2015;2015:190-6.

73 Tamamyan GN, Kantarjian HM, Ning J, et al. MalignancyAssociated hemophagocytic lymphohistiocytosis in adults: relation to hemophagocytosis, characteristics, and outcomes. Cancer 2016;122:2857-66.

74 Rutkowska-Zapała M, Suski M, Szatanek R, et al. Human monocyte subsets exhibit divergent angiotensin I-converting activity. Clin Exp Immunol 2015;181:126-32.

75 Liu Y, Chen D, Hou J, et al. An inter-correlated cytokine network identified at the center of cytokine storm predicted COVID-19 prognosis. Cytokine 2021;138:155365.

76 Aziz M, Fatima R, Assaly R. Elevated interleukin-6 and severe COVID-19: a meta-analysis. J Med Virol 2020;92:2283-5.

77 Manson JJ, Crooks C, Naja M, et al. COVID-19-associated hyperinflammation and escalation of patient care: a retrospective longitudinal cohort study. The Lancet Rheumatology 2020;2:e594-602 
78 Webb BJ, Peltan ID, Jensen P, et al. Clinical criteria for COVID19-associated hyperinflammatory syndrome: a cohort study. The Lancet Rheumatology 2020;2:e754-63.

79 Fardet L, Galicier L, Lambotte O, et al. Development and validation of the HScore, a score for the diagnosis of reactive hemophagocytic syndrome. Arthritis \& Rheumatology 2014;66:2613-20.

80 Yoshikawa T, Hill T, Li K, et al. Severe acute respiratory syndrome (SARS) coronavirus-induced lung epithelial cytokines exacerbate SARS pathogenesis by modulating intrinsic functions of monocytederived macrophages and dendritic cells. J Virol 2009;83:3039-48.

81 Wang W, Ye L, Ye L, Li B, et al. Up-Regulation of IL-6 and TNF- $\alpha$ induced by SARS-coronavirus spike protein in murine macrophages via NF-kB pathway. Virus Res 2007;128:1-8.

82 Bedin A-S, Makinson A, Picot M-C, et al. Monocyte CD169 expression as a biomarker in the early diagnosis of coronavirus disease 2019. J Infect Dis 2021;223:562-7.

83 Zhou Y, Fu B, Zheng X, et al. Pathogenic T-cells and inflammatory monocytes incite inflammatory storms in severe COVID-19 patients. National Science Review 2020;7:998-1002.

84 Tay MZ, Poh CM, Rénia L, et al. The trinity of COVID-19: immunity, inflammation and intervention. Nat Rev Immunol 2020;20:363-74.

85 Huang J, Hume AJ, Abo KM, et al. SARS-CoV-2 infection of pluripotent stem cell-derived human lung alveolar type 2 cells elicits a rapid Epithelial-Intrinsic inflammatory response. Cell Stem Cell 2020;27:962-73.

86 Cheng MH, Zhang S, Porritt RA, et al. Superantigenic character of an insert unique to SARS-CoV-2 spike supported by skewed TCR repertoire in patients with hyperinflammation. Proc Natl Acad Sci U S A 2020;117:25254-62.

87 Scaglioni V, Soriano ER. Are superantigens the cause of cytokine storm and viral sepsis in severe COVID-19? observations and hypothesis. Scand J Immunol 2020;92:e12944.

88 Norelli M, Camisa B, Barbiera G, et al. Monocyte-Derived IL-1 and IL-6 are differentially required for cytokine-release syndrome and neurotoxicity due to CAR T cells. Nat Med 2018;24:739-48.

89 Crestani B, Cornillet P, Dehoux M, et al. Alveolar type II epithelial cells produce interleukin- 6 in vitro and in vivo. Regulation by alveolar macrophage secretory products. J. Clin. Invest. 1994;94:731-40.

90 Jafarzadeh A, Chauhan P, Saha B, et al. Contribution of monocytes and macrophages to the local tissue inflammation and cytokine storm in COVID-19: lessons from SARS and MERS, and potential therapeutic interventions. Life Sci 2020;257:118102.

91 Banu N, Panikar SS, Leal LR, et al. Protective role of ACE2 and its downregulation in SARS-CoV-2 infection leading to macrophage activation syndrome: therapeutic implications. Life Sci 2020;256:117905.

92 Shantsila E, Lip GYH. Monocytes in acute coronary syndromes. Arterioscler Thromb Vasc Biol 2009;29:1433-8.

93 Suzuki Y, Ruiz-Ortega M, Lorenzo O, et al. Inflammation and angiotensin II. Int J Biochem Cell Biol 2003:35:881-900.

94 Liu F, Xu A, Zhang Y, et al. Patients of COVID-19 may benefit from sustained Lopinavir-combined regimen and the increase of eosinophil may predict the outcome of COVID-19 progression. Int $J$ of Dis 2020;95:183-91.

95 Qin C, Zhou L, Hu Z, et al. Dysregulation of immune response in patients with coronavirus 2019 (COVID-19) in Wuhan, China. Clin Infect Dis 2020;71:762-768.

96 Montesarchio V, Parella R, lommelli C, et al. Outcomes and biomarker analyses among patients with COVID-19 treated with interleukin 6 (IL-6) receptor antagonist sarilumab at a single institution in Italy. J Immunother Cancer 2020;8:e001089.

97 Bialas A, Kornicki K, Ciebiada M, et al. Miłkowska - Dymanowska J, Piotrowski W, et al. Eosinopenia as a prognostic factor in patients with acute exacerbation of chronic obstructive pulmonary disease. European Respiratory Journal 2017;50:PA2110.

98 Eng SS, DeFelice ML. The role and immunobiology of eosinophils in the respiratory system: a comprehensive review. Clin Rev Allergy Immunol 2016;50:140-58.

99 Isobe $Y$, Kato T, Arita M. Emerging roles of eosinophils and eosinophil-derived lipid mediators in the resolution of inflammation. Front Immunol 2012;3.

100 Ruan Q, Yang K, Wang W, et al. Clinical predictors of mortality due to COVID-19 based on an analysis of data of 150 patients from Wuhan, China. Intensive Care Med 2020;46:846-8.

101 Tanaka T, Narazaki M, Kishimoto T. II-6 in inflammation, immunity, and disease. Cold Spring Harb Perspect Biol 2014;6:a016295

102 Uciechowski P, Dempke WCM. Interleukin-6: a Masterplayer in the cytokine network. Oncology 2020;98:131-7.

103 Wolf J, Rose-John S, Garbers C. Interleukin-6 and its receptors: highly regulated and dynamic system. Cytokine 2014;70:11-20.
104 Okabayashi T, Kariwa H, Yokota S-ichi, Si Y, et al. Cytokine regulation in SARS coronavirus infection compared to other respiratory virus infections. J Med Virol 2006;78:417-24.

105 Eskilsson A, Mirrasekhian E, Dufour S, et al. Immune-Induced fever is mediated by IL-6 receptors on brain endothelial cells coupled to STAT3-dependent induction of brain endothelial prostaglandin synthesis. J Neurosci 2014;34:15957-61.

106 Schumacher N, Meyer D, Mauermann A, et al. Shedding of endogenous interleukin-6 receptor (IL-6R) is governed by a disintegrin and metalloproteinase (ADAM) proteases while a fulllength IL-6R isoform localizes to circulating microvesicles. $J$ of Chem 2015;290:26059-71.

107 Schumacher N, Rose-John S. Adam17 activity and IL-6 transsignaling in inflammation and cancer. Cancers 2019;11:1736.

108 Puzanov I, Diab A, Abdallah K, et al. Managing toxicities associated with immune checkpoint inhibitors: consensus recommendations from the Society for immunotherapy of cancer (SITC) toxicity management Working group. J Immunother Cancer 2017;5:95.

109 Le RQ LL, Yuan W, Shord SS, et al. Fda approval summary: tocilizumab for treatment of chimeric antigen receptor T cellinduced severe or life-threatening cytokine release syndrome. The oncologist 2018;23:943-7.

110 Stroud CRG, Hegde A, Cherry C, et al. Tocilizumab for the management of immune mediated adverse events secondary to PD-1 blockade. Journal of Oncology Pharmacy Practice 2019;25:551-7.

111 Ikonomidis I, Pavlidis G, Katsimbri P, et al. Tocilizumab improves oxidative stress and endothelial glycocalyx: a mechanism that may explain the effects of biological treatment on COVID-19. Food and Chemical Toxicology 2020;145:111694.

112 Liu F, Li L, Xu M, et al. Prognostic value of interleukin-6, C-reactive protein, and procalcitonin in patients with COVID-19. Journal of Clinical Virology 2020;127:104370.

113 Zhang L, Zhu F, Xie L, et al. Clinical characteristics of COVID19-infected cancer patients: a retrospective case study in three hospitals within Wuhan, China. Annals of Oncology 2020;31:894-901.

114 Luo P, Liu Y, Qiu L, et al. Tocilizumab treatment in COVID-19: a single center experience. J Med Virol 2020;92:814-8.

115 Cascella M, Mauro I, De Blasio E, et al. Rapid and impressive response to a combined treatment with single-dose tocilizumab and NIV in a patient with COVID-19 Pneumonia/ARDS. Medicina 2020;56:377.

116 Perrone F, Piccirillo MC, Ascierto PA, et al. Tocilizumab for patients with COVID-19 pneumonia. The single-arm TOCIVID-19 prospective trial. J Trans/ Med 2020;18:405.

117 Fernández-Ruiz M, López-Medrano F, Pérez-Jacoiste Asín MA, et al. Tocilizumab for the treatment of adult patients with severe COVID-19 pneumonia: a single-center cohort study. J Med Virol 2020.

118 Toniati P, Piva S, Cattalini M, et al. Tocilizumab for the treatment of severe COVID-19 pneumonia with hyperinflammatory syndrome and acute respiratory failure: a single center study of 100 patients in Brescia, Italy. Autoimmun Rev 2020;19:102568.

119 Della-Torre E, Campochiaro C, Cavalli G, et al. Interleukin-6 blockade with sarilumab in severe COVID-19 pneumonia with systemic hyperinflammation: an open-label cohort study. Ann Rheum Dis 2020;79:1277-85.

120 Regeneron Pharmaceuticals Inc. Regeneron and Sanofi provide update on Kevzara ${ }^{\circledR}$ (sarilumab) phase 3 US. trial in COVID-19 patients, 2020. Available: https://newsroom.regeneron.com/ news-releases/news-release-details/regeneron-and-sanofi-provideupdate-kevzarar-sarilumab-phase-3

121 Hermine O, Mariette X, Tharaux P-L, et al. Effect of tocilizumab vs usual care in adults hospitalized with COVID-19 and moderate or severe pneumonia. JAMA Intern Med 2021;181:32-40.

122 Marshall JC, Murthy S, Diaz J, et al. A minimal common outcome measure set for COVID-19 clinical research. Lancet Infect Dis 2020;20:e192-7.

123 Salama C, Han J, Yau L, et al. Tocilizumab in nonventilated patients hospitalized with Covid-19 pneumonia. medRxiv 2020.

124 , Gordon AC, Mouncey PR, et al, REMAP-CAP Investigators Interleukin-6 receptor antagonists in critically ill patients with Covid-19. N Engl J Med 2021. doi:10.1056/NEJMoa2100433. [Epub ahead of print: 25 Feb 2021].

125 Horby PW, Pessoa-Amorim G, Peto L, et al. Tocilizumab in patients admitted to hospital with COVID-19 (recovery): preliminary results of a randomised, controlled, open-label, platform trial. medRxiv 2021.

126 Gupta S, Wang W, Hayek SS, et al. Association between early treatment with tocilizumab and mortality among critically ill patients with COVID-19. JAMA Intern Med 2021:181:41. 
127 Rosas I, Bräu N, Waters M, et al. Tocilizumab in hospitalized patients with COVID-19 pneumonia. medRxiv 2020.

128 Stone JH, Frigault MJ, Serling-Boyd NJ, et al. Efficacy of tocilizumab in patients hospitalized with Covid-19. N Engl J Med 2020;383:2333-44

129 Veiga VC, Prats J, Farias DLC, et al. Effect of tocilizumab on clinical outcomes at 15 days in patients with severe or critical coronavirus disease 2019: randomised controlled trial. BMJ 2021;372:n84.

130 Aziz M, Haghbin H, Abu Sitta E, et al. Efficacy of tocilizumab in COVID-19: a systematic review and meta-analysis. J Med Virol 2021;93:1620-30

131 Malgie J, Schoones JW, Pijls BG. Decreased mortality in coronavirus disease 2019 patients treated with tocilizumab: a rapid systematic review and meta-analysis of observational studies. Clinical Infectious Diseases 2020;20.

132 Tleyjeh IM, Kashour Z, Damlaj M, et al. Efficacy and safety of tocilizumab in COVID-19 patients: a living systematic review and meta-analysis. Clinical Microbiology and Infection 2021;27:215-27.

133 Beigel JH, Tomashek KM, Dodd LE, et al. Remdesivir for the treatment of Covid-19 - final report. N Engl J Med 2020;383:1813-26.

134 Rochwerg B, Agoritsas T, Lamontagne F, et al. A living who guideline on drugs for covid-19. BMJ 2020;370:m3379.

135 B A, RL M, AH S VL. Infectious diseases Society of America guidelines on the treatment and management of patients with COVID-19: IDSA, 2021. Available: https://www.idsociety. org/practice-guideline/covid-19-guideline-treatment-andmanagement/\#toc-8

136 Horby P, Lim WS, Emberson JR. Dexamethasone in hospitalized patients with Covid-19 - preliminary report. $N$ Engl J Med 2020;384:693-704. doi:10.1056/NEJMoa2021436

137 Salvarani C, Dolci G, Massari M, et al. Effect of tocilizumab vs standard care on clinical worsening in patients hospitalized with COVID-19 pneumonia: a randomized clinical trial. JAMA Intern Med 2021;181:24-31.

138 Ramiro S, Mostard RLM, Magro-Checa C, et al. Historically controlled comparison of glucocorticoids with or without tocilizumab versus supportive care only in patients with COVID-19associated cytokine storm syndrome: results of the chiC study. Ann Rheum Dis 2020;79:1143-51.

139 Martínez-Urbistondo D, Costa Segovia R, Suárez Del Villar Carrero $\mathrm{R}$, et al. Early combination of tocilizumab and corticosteroids: an upgrade in anti-inflammatory therapy for severe COVID. Clin Infect Dis 2020. doi:10.1093/cid/ciaa910. [Epub ahead of print: 04 Jul 2020].

140 Rhodes JM, Subramanian S, Laird E, et al. Editorial: low population mortality from COVID-19 in countries South of latitude 35 degrees North supports vitamin $\mathrm{D}$ as a factor determining severity. Aliment Pharmacol Ther 2020;51:1434-7.

141 Dhar D, Mohanty A. Gut microbiota and Covid-19- possible link and implications. Virus Res 2020;285:198018.

142 Zuo T, Zhan H, Zhang F, et al. Alterations in fecal fungal microbiome of patients with COVID-19 during time of hospitalization until discharge. Gastroenterology 2020;159:1302-10.
143 Zuo T, Liu Q, Zhang F, et al. Depicting SARS-CoV-2 faecal viral activity in association with gut microbiota composition in patients with COVID-19. Gut 2021;70:276-84.

144 Kuhn KA, Schulz HM, Regner EH, et al. Bacteroidales recruit IL6 -producing intraepithelial lymphocytes in the colon to promote barrier integrity. Mucosal Immunol 2018;11:357-68.

145 Luu M, Weigand K, Wedi F, et al. Regulation of the effector function of CD8. Sci Rep 2018;8:14430.

146 Islam N, Khunti K, Dambha-Miller H, et al. COVID-19 mortality: a complex interplay of sex, gender and ethnicity. Eur J Public Health 2020;30:847-8.

147 B A, RL M, AH S VL, et al. Infectious Diseases Society of America guidelines on the treatment and management of patients with COVID-19: IDSA, 2021. Available: https://www.idsociety. org/practice-guideline/covid-19-guideline-treatment-andmanagement/\#toc-8

148 Hunter CA, Jones SA. II-6 as a keystone cytokine in health and disease. Nat Immunol 2015;16:448-57.

149 Potere N, Di Nisio M, Rizzo G, et al. Low-Dose subcutaneous tocilizumab to prevent disease progression in patients with moderate COVID-19 pneumonia and hyperinflammation. Int $J$ of Dis 2020;100:421-4.

150 Busani S, Bedini A, Biagioni E, et al. Two fatal cases of acute liver failure due to HSV-1 infection in COVID-19 patients following immunomodulatory therapies. Clin Infect Dis 2020.

151 Guaraldi G, Meschiari M, Cozzi-Lepri A, et al. Tocilizumab in patients with severe COVID-19: a retrospective cohort study. Lancet Rheumatol 2020;2:e474-84.

152 Somers EC, Eschenauer GA, Troost JP, et al. Tocilizumab for treatment of mechanically ventilated patients with COVID-19. Clin Infect Dis 2020;382.

153 Arnaldez FI, O'Day SJ, Drake CG, et al. The Society for immunotherapy of cancer perspective on regulation of interleukin-6 signaling in COVID-19-related systemic inflammatory response. Journal for ImmunoTherapy of Cancer 2020;8:e000930.

154 Roivant announces development of anti-gm-csf monoclonal antibody to prevent and treat acute respiratory distress syndrome (ARDS) in patients with COVID-19 - Roivant Sciences, 2020. Available: https://roivant.com/roivant-announces-developmentof-anti-gm-csf-monoclonal-antibody-to-prevent-and-treat-acuterespiratory-distress-syndrome-ards-in-patients-with-covid-19.html

155 Huet T, Beaussier H, Voisin O, et al. Anakinra for severe forms of COVID-19: a cohort study. The Lancet Rheumatology 2020;2:e393-400.

156 Iglesias-Julián E, López-Veloso M, de-la-Torre-Ferrera N, et al. High dose subcutaneous Anakinra to treat acute respiratory distress syndrome secondary to cytokine storm syndrome among severely ill COVID-19 patients. J Autoimmun 2020;115:102537.

157 Cingolani A, Tummolo AM, Montemurro G, et al. Baricitinib as rescue therapy in a patient with COVID-19 with no complete response to sarilumab. Infection 2020;48:767-71.

158 Kalil AC, Patterson TF, Mehta AK, et al. Baricitinib plus Remdesivir for hospitalized adults with Covid-19. N Engl J Med 2021;384:795-807. 